\title{
SIMPLICIAL MONOIDS AND SEGAL CATEGORIES
}

\author{
JULIA E. BERGNER
}

\begin{abstract}
Much research has been done on structures equivalent to topological or simplicial groups. In this paper, we consider instead simplicial monoids. In particular, we show that the usual model category structure on the category of simplicial monoids is Quillen equivalent to an appropriate model category structure on the category of simplicial spaces with a single point in degree zero. In this second model structure, the fibrant objects are reduced Segal categories. We then generalize the proof to relate simplicial categories with a fixed object set to Segal categories with the same fixed set in degree zero.
\end{abstract}

\section{INTRODUCTION}

There has been much work done showing the equivalences of topological (or simplicial) groups, group-like $A_{\infty}$-spaces, and loop spaces. References for this work include [22], 23], and the more recent [1] and [2]. In this paper, we show that there are some analogous comparisons to be made when we work with simplicial monoids rather than simplicial groups. We show that, from the perspective of homotopy theory, simplicial monoids are essentially the same as reduced Segal categories (Definition [1.2).

In order to describe the problem more fully, we begin with some notation and terminology. Let $\boldsymbol{\Delta}$ denote the cosimplicial category, or category whose objects are finite ordered sets $[n]=\{0, \ldots, n\}$ for $n \geq 0$ and whose morphisms are orderpreserving maps between them. Then $\boldsymbol{\Delta}^{o p}$ is the opposite of this category and is called the simplicial category. Recall that a simplicial set $X$ is a functor $\boldsymbol{\Delta}^{o p} \rightarrow$ Sets. We will denote the category of simplicial sets by SSets. (In the course of this paper we will sometimes refer to simplicial sets as spaces, due to their homotopytheoretic similarity with topological spaces [15] 3.6.7].) Induced from the maps in $\boldsymbol{\Delta}^{o p}$ are the face maps $d_{i}: X_{n} \rightarrow X_{n-1}$ and degeneracy maps $s_{i}: X_{n} \rightarrow X_{n-1}$. A few of the simplicial sets we will use are the $n$-simplex $\Delta[n]$ for each $n \geq 0$, its boundary $\dot{\Delta}[n]$, and the boundary with the $k$ th face removed, $V[n, k]$. More details about simplicial sets can be found in [12, I]. We denote by $|X|$ the topological space given by geometric realization of the simplicial set $X$ [12, I.2].

More generally, a simplicial object in a category $\mathcal{C}$ is a functor $\boldsymbol{\Delta}^{o p} \rightarrow \mathcal{C}$. In particular, a functor $\boldsymbol{\Delta}^{o p} \rightarrow$ SSets is a simplicial space or bisimplicial set [12, IV]. Given a simplicial set $X$, we will also use $X$ to denote the constant simplicial space with the simplicial set $X$ in each degree. By $X^{t}$ (which should be thought of as " $X$-transposed") we will denote the simplicial space such that $\left(X^{t}\right)_{n}$ is the constant

Date: February 8, 2020.

2000 Mathematics Subject Classification. Primary 18G30; Secondary 18E35, 18C10, 55 U40.

Key words and phrases. simplicial monoids, Segal categories, simplicial categories, model categories. 
simplicial set $X_{n}$, or the simplicial set which has the set $X_{n}$ at each level. We will denote the category of all simplicial spaces by $\mathcal{S S e t s}^{\boldsymbol{\Delta}^{o p}}$.

In this paper, we consider a very specific kind of simplicial space, namely, a reduced Segal category. We first consider the more basic definition of a Segal precategory.

Definition 1.1. A Segal precategory is a simplicial space $X$ such that $X_{0}$ is a discrete simplicial set. If $X_{0}$ consists of a single point, then we will call $X$ a reduced Segal precategory.

Now note that for any simplicial space $X$ there is a Segal map

$$
\varphi_{k}: X_{k} \rightarrow \underbrace{X_{1} \times_{X_{0}} \ldots \times_{X_{0}} X_{1}}_{k}
$$

for each $k \geq 2$. (The precise definition of this map is given in section 2.)

Definition 1.2. [14, §2] A Segal category $X$ is a Segal precategory such that the Segal map $\varphi_{k}$ is a weak equivalence of simplicial sets for each $k \geq 2$. A reduced Segal category is a Segal category such that $X_{0}$ consists of a single point.

Now, let $M$ be a simplicial monoid, by which we mean a simplicial object in the category $\mathcal{M}$ on of monoids, or a functor $\boldsymbol{\Delta}^{o p} \rightarrow \mathcal{M}$ on. We will use an alternate viewpoint in which we use algebraic theories to define simplicial monoids. We begin with the definition of an algebraic theory. Some references for algebraic theories include chapter 3 of [6], the introduction to [1], and section 3 of [4].

Definition 1.3. An algebraic theory $\mathcal{T}$ is a small category with finite products and objects denoted $T_{n}$ for $n \geq 0$. For each $n, T_{n}$ is equipped with an isomorphism $T_{n} \cong\left(T_{1}\right)^{n}$. Note in particular that $T_{0}$ is the terminal object in $T$.

Here we will consider one particular theory, the theory of monoids, which we denote $\mathcal{T}_{M}$. To describe this theory, we first consider the full subcategory of the category of monoids whose objects $T_{n}$ are the isomorphism classes of free monoids on $n$ generators. We then define the theory of monoids $\mathcal{T}_{M}$ to be the opposite of this category. Thus $T_{n}$, which is canonically the coproduct of $n$ copies of $T_{1}$ in $\mathcal{M}$ on, becomes the product of $n$ copies of $T_{1}$ in $\mathcal{T}_{M}$. It follows that there is a projection map $p_{i}: T_{n} \rightarrow T_{1}$ for each $i \geq 2$ in addition to other monoid maps. In fact, there are such projection maps in any algebraic theory. We use them to make the following definition.

Definition 1.4. [1, 1.1] Given an algebraic theory $\mathcal{T}$, a strict simplicial $\mathcal{T}$-algebra (or simply $\mathcal{T}$-algebra) $A$ is a product-preserving functor $A: \mathcal{T} \rightarrow$ SSets. Here, "product-preserving" means that for each $n \geq 0$ the canonical map

$$
A\left(T_{n}\right) \rightarrow A\left(T_{1}\right)^{n},
$$

induced by the $n$ projection maps $T_{n} \rightarrow T_{1}$, is an isomorphism of simplicial sets. In particular, $A\left(T_{0}\right)$ is the one-point simplicial set $\Delta[0]$.

In general, a $\mathcal{T}$-algebra $A$ defines a strict algebraic structure on the space $A\left(T_{1}\right)$ corresponding to the theory $\mathcal{T}[1, \S 1]$. So, a $\mathcal{T}_{M}$-algebra $A$ defines a monoid structure on the space $A\left(T_{1}\right)$. It turns out that the category of simplicial monoids is equivalent

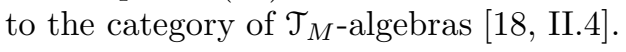

We can also consider the case where the products are not preserved strictly, but only up to homotopy. 
Definition 1.5. 1, 1.2] Given an algebraic theory $\mathcal{T}$, a homotopy $\mathcal{T}$-algebra is a functor $X: \mathcal{T} \rightarrow$ SSets which preserves products up to homotopy. The functor $X$ preserves products up to homotopy if, for each $n \geq 0$ the canonical map

$$
X\left(T_{n}\right) \rightarrow X\left(T_{1}\right)^{n}
$$

induced by the projection maps $p_{i}: T_{n} \rightarrow T_{1}$ is a weak equivalence of simplicial sets. In particular, we assume that $X\left(T_{0}\right)$ is weakly equivalent to $\Delta[0]$.

Now that we have established our definitions for simplicial monoids, we turn to reduced Segal categories. As with the simplicial monoids, we would like to think of our reduced Segal categories as objects in a category of diagrams of a particular shape. In this case our diagram will be the simplicial category $\boldsymbol{\Delta}^{o p}$. Because $\boldsymbol{\Delta}^{o p}$ is not an algebraic theory, and in particular because $[n]$ is not isomorphic to the product of $n$ copies of [1], we cannot use the same terminology of algebras as we did for the theories.

We first define $n$ specified maps $[1] \rightarrow[n]$ in $\boldsymbol{\Delta}$. Let $\alpha^{i}:[1] \rightarrow[n]$ be the map such that $\alpha^{i}(0)=i$ and $\alpha^{i}(1)=i+1$, defined for each $0 \leq i \leq n-1$. Using these maps, we will require the following product property on the diagrams:

Definition 1.6. A functor $F: \Delta^{o p} \rightarrow$ SSets is special if $F([0])=\Delta[0]$ and if the canonical map

induced by the map

$$
F([n]) \stackrel{\simeq}{\longrightarrow} F([1])^{n}
$$

$$
\coprod_{0 \leq i \leq n-1}\left(\alpha^{i}:[1] \rightarrow[n]\right)
$$

is a weak equivalence of simplicial sets.

Thus, a reduced Segal category is a special functor $F: \boldsymbol{\Delta}^{o p} \rightarrow$ SSets. Note that unlike in the case of homotopy $\mathcal{T}$-algebras, we actually require $F([0])$ to be $\Delta[0]$, so that we actually get a reduced Segal category and not just a simplicial space equivalent to one.

The precise relationship that we would like to prove between the simplicial monoids (or $\mathcal{T}_{M}$-algebras) and Segal categories is a Quillen equivalence of model categories. (We will give a brief overview of model categories and Quillen equivalences in the next section.)

In this paper, we will prove the existence of a model category structure $\mathcal{A l g} g^{\mathcal{T}_{M}}$ on the category of $\mathcal{T}_{M}$-algebras. There is another model category structure $\mathcal{L} \mathcal{S} \mathcal{S} p_{*, f}$ on the category of reduced Segal precategories such that the fibrant objects are reduced Segal categories. (We cannot obtain a model category structure on the category of reduced Segal categories because this category is not closed under limits and colimits.) Our main result is the following theorem:

Theorem 1.7. The model category structure $\mathcal{A l g}^{\mathcal{T}_{M}}$ is Quillen equivalent to the model category structure $\mathcal{L} \mathcal{S} S p_{*, f}$.

We prove this theorem in section 4 after reviewing model categories in section 2 and setting up our particular model category structures in section 3 . In section 5 , we generalize this result to an analogous statement for simplicial categories with a fixed object set $\mathcal{O}$ and Segal categories with the same fixed set $\mathcal{O}$ in degree zero. In [5], we prove the more general result that there is a Quillen equivalence between a model category structure on the category of all small simplicial categories (where 
the weak equivalences are a simplicial version of equivalences of categories [3, §1]) and a model category structure on the category of all Segal precategories (with analogous weak equivalences).

The techniques of this paper should carry over to a similar result for simplicial groups by modifying the category $\boldsymbol{\Delta}^{o p}$. Let $I[n]$ denote the category with $n+1$ objects and exactly one isomorphism between any two objects [19, §6]. Analogously to the way we form $\boldsymbol{\Delta}$ from the categories $[n]$, we define the category $\mathbf{I} \boldsymbol{\Delta}$ whose objects are the $I[n]$ for all $n \geq 0$ and whose morphisms are order-preserving maps. (Here "order-preserving" should be taken to mean "just like the maps in $\boldsymbol{\Delta}$," since the ordering of objects in $I[n]$ is less clear than in $[n]$.)

Now, a special functor $\mathbf{I} \boldsymbol{\Delta}^{o p} \rightarrow$ SSets is a reduced Segal groupoid. We can then consider the theory of groups $\mathcal{T}_{G}$ and strict $\mathcal{T}_{G}$-algebras, which are essentially simplicial groups. The arguments of this paper should give us the result that the model category structure on $\mathcal{T}_{G}$-algebras is Quillen equivalent to an appropriate model structure on "reduced Segal pregroupoids." The explicit argument will be included in a later paper.

The idea here, for both monoids and groups, is that when we consider diagrams of spaces given by a particular algebraic theory, from a homotopy-theoretic perspective we can actually consider diagrams of spaces given by some simpler, pared-down diagram in the sense that $\boldsymbol{\Delta}^{o p}$ can be considered a simplification of $\mathcal{T}_{M}$. Similar work has been done by Segal 21] for groups and abelian monoids, and by Bousfield 7] for groups and $n$-fold loop spaces. We hope to find more such examples in the future.

Acknowledgments. I am grateful to Bill Dwyer for many helpful conversations about this paper.

\section{A Review of Model Categories}

Recall that a model category structure on a category $\mathcal{C}$ is a choice of three distinguished classes of morphisms: fibrations, cofibrations, and weak equivalences. A (co)fibration which is also a weak equivalence will be called an acyclic (co)fibration. With this choice of three classes of morphisms, $\mathcal{C}$ is required to satisfy five axioms MC1-MC5 [11, 3.3].

An object $X$ in $\mathcal{C}$ is fibrant if the unique map $X \rightarrow *$ from $X$ to the terminal object is a fibration. Dually, $X$ is cofibrant if the unique map $\phi \rightarrow X$ from the initial object to $X$ is a cofibration. The factorization axiom (MC5) guarantees that each object $X$ has a weakly equivalent fibrant replacement $\widehat{X}$ and a weakly equivalent cofibrant replacement $\widetilde{X}$. These replacements are not necessarily unique, but they can be chosen to be functorial in the cases we will use [15, 1.1.3].

The model category structures which we will discuss are all cofibrantly generated. A cofibrantly generated model category $\mathcal{C}$ is a model category for which there are two sets of morphisms, one of generating cofibrations and one of generating acyclic cofibrations, such that a map is a fibration if and only if it has the right lifting property with respect to the generating acyclic cofibrations, and a map is an acyclic fibration if and only if it has the right lifting property with respect to the generating cofibrations [13.11.1.2]. (Recall that if the dotted arrow lift exists in the solid arrow 
diagram

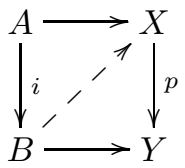

then we say that $i$ has the left lifting property with respect to $p$ and that $p$ has the right lifting property with respect to $i$.) To state the conditions we will use to determine when we have a cofibrantly generated model category structure, we make the following definition.

Definition 2.1. [13, 10.5.2] Let $\mathcal{C}$ be a category and $I$ a set of maps in $\mathcal{C}$. Then an $I$-injective is a map which has the right lifting property with respect to every map in $I$. An $I$-cofibration is a map with the left lifting property with respect to every $I$-injective.

Using this definition, we will use the following theorem to determine when we have a cofibrantly generated model category structure.

Theorem 2.2. 13, 11.3.1] Let $\mathcal{M}$ be a category which has all finite limits and colimits. Suppose that $\mathcal{M}$ has a class of weak equivalences which satisfies the two-outof-three property (model category axiom MC2) and which is closed under retracts. Let $I$ and $J$ be sets of maps in $\mathcal{M}$ which satisfy the following conditions:

(1) Both $I$ and J permit the small object argument [13 10.5.15].

(2) Every $J$-cofibration is an I-cofibration and a weak equivalence.

(3) Every I-injective is a J-injective and a weak equivalence.

(4) One of the following conditions holds:

(i) A map that is an I-cofibration and a weak equivalence is a J-cofibration, or

(ii) A map that is both a J-injective and a weak equivalence is an Iinjective.

Then there is a cofibrantly generated model category structure on $\mathcal{M}$ in which $I$ is a set of generating cofibrations and $J$ is a set of generating acyclic cofibrations.

We will refer to the standard model category structure on the category SSets of simplicial sets. In this case, a weak equivalence is a map of simplicial sets $f: X \rightarrow Y$ such that the induced map $|f|:|X| \rightarrow|Y|$ is a weak homotopy equivalence of topological spaces. The cofibrations are monomorphisms, and the fibrations are the maps with the right lifting property with respect to the acyclic cofibrations [12, I.11.3]. This model category structure is cofibrantly generated; the generating cofibrations are the maps $\dot{\Delta}[n] \rightarrow \Delta[n]$ for $n \geq 0$, and the generating acyclic cofibrations are the maps $V[n, k] \rightarrow \Delta[n]$ for $n \geq 1$ and $0 \leq k \leq n$.

Each model structure in this paper will have the additional structure of a simplicial model category. A simplicial category is a category with a simplicial set of morphisms between any two objects. A simplicial model category, then, is a model category $\mathcal{M}$ which is also a simplicial category, satisfying some axioms 13 , 9.1.6]. (Notice that this terminology is somewhat confusing in that simplicial objects in the category of categories are not necessarily simplicial categories, nor are simplicial objects in the category of model categories actually simplicial model categories, at least not without imposing further conditions.) The important part of this structure is that it enables us to talk about the function complex, or simplicial 
set $\operatorname{Map}(X, Y)$ for any pair of objects $X$ and $Y$ in $\mathcal{M}$. For example, the model category SSets is a simplicial model category. For simplicial sets $X$ and $Y$, the simplicial set $\operatorname{Map}(X, Y)$ is given by

$$
\operatorname{Map}(X, Y)_{n}=\operatorname{Hom}(X \times \Delta[n], Y) .
$$

However, a function complex is not necessarily homotopy invariant, so we have the following definition.

Definition 2.3. 13, 17.3.1] If $\mathcal{M}$ is a simplicial model category, then the homotopy function complex $\operatorname{Map}^{h}(X, Y)$ is given by $\operatorname{Map}(\widetilde{X}, \widehat{Y})$, where $\widetilde{X}$ is a cofibrant replacement of $X$ in $\mathcal{M}$ and $\widehat{Y}$ is a fibrant replacement for $Y$.

More generally, a homotopy function complex $\operatorname{Map}^{h}(X, Y)$ in a (not necessarily simplicial) model category $\mathcal{M}$, or in a category with weak equivalences, is the simplicial set $\operatorname{Map}(X, Y)$ given by the simplicial set of morphisms between $X$ and $Y$ in the hammock localization $L^{H} \mathcal{M}$ of $\mathcal{M} 9$.

Many of the model category structures that we will work with will be obtained by localizing a given model category structure with respect to a set of maps. To make sense of this notion, we make the following definitions.

Definition 2.4. Suppose that $S=\{f: A \rightarrow B\}$ is a set of maps in a model category $\mathcal{M}$. An $S$-local object $X$ is a fibrant object of $\mathcal{M}$ such that for any $f$ : $A \rightarrow B$ in $S$, the induced map on homotopy function complexes

$$
f^{*}: \operatorname{Map}^{h}(B, W) \rightarrow \operatorname{Map}^{h}(A, W)
$$

is a weak equivalence of simplicial sets. A map $g: X \rightarrow Y$ in $\mathcal{M}$ is then an $S$-local equivalence if for every local object $W$, the induced map on homotopy function complexes

$$
g^{*}: \operatorname{Map}^{h}(Y, W) \rightarrow \operatorname{Map}^{h}(X, W)
$$

is a weak equivalence of simplicial sets.

We now define the localization of a model category $\mathcal{M}$. The following theorem holds for all model categories $\mathcal{M}$ which are left proper and cellular. We will not define these properties here but refer the reader to [13 13.1.1, 12.1.1] for details. All the model categories we will work with can be shown to satisfy these properties.

Theorem 2.5. 13, 4.1.1] Let $\mathcal{M}$ be a left proper cellular model category. There is a model category structure $\mathcal{L}_{S} \mathcal{M}$ on the underlying category of $\mathcal{M}$ such that:

(1) The weak equivalences are the $S$-local equivalences.

(2) The cofibrations are precisely the cofibrations of $\mathcal{M}$.

(3) The fibrations are the maps which have the right lifting property with respect to the maps which are both cofibrations and $S$-local equivalences.

(4) The fibrant objects are the S-local objects.

(5) If $\mathcal{M}$ is a simplicial model category, then its simplicial structure induces a simplicial structure on $\mathcal{L}_{S} \mathcal{M}$.

In particular, given an object $X$ of $\mathcal{M}$, we can talk about its functorial fibrant replacement $L X$ in $\mathcal{L}_{S} \mathcal{M}$. The object $L X$ is an $S$-local object which is fibrant in $\mathcal{M}$, and we will call it the localization of $X$ in $\mathcal{L}_{S} \mathcal{M}$.

We now state the definition of a Quillen pair of model category structures. Recall that for categories $\mathcal{C}$ and $\mathcal{D}$ a pair of functors

$$
F: \mathcal{C} \rightleftarrows \mathcal{D}: R
$$


is an adjoint pair if for each object $X$ of $\mathcal{C}$ and object $Y$ of $\mathcal{D}$ there is an isomorphism $\varphi: \operatorname{Hom}_{\mathcal{D}}(F X, Y) \rightarrow \operatorname{Hom}_{\mathcal{C}}(X, R Y)$ [16, IV.1]. The adjoint pair is sometimes written as the triple $(F, R, \varphi)$.

Definition 2.6. [15, 1.3.1] If $\mathcal{C}$ and $\mathcal{D}$ are model categories, then an adjoint pair $(F, R, \varphi)$ between them is a Quillen pair if one of the following equivalent statements holds:

(1) $F$ preserves cofibrations and acyclic cofibrations.

(2) $R$ preserves fibrations and acyclic fibrations.

We will use the following theorem to show that we have a Quillen pair of localized model category structures.

Theorem 2.7. 13, 3.3.20] Let $\mathcal{C}$ and $\mathcal{D}$ be left proper, cellular model categories and let $(F, R, \varphi)$ be a Quillen pair between them. Let $S$ be a set of maps in $\mathcal{C}$ and $L_{S} \mathcal{C}$ the localization of $\mathcal{C}$ with respect to $S$. Then if $\mathbf{L} F S$ is the set in $\mathcal{D}$ obtained by applying the left derived functor of $F$ to the set $S$ [13 8.5.11], then $(F, R, \varphi)$ is also a Quillen pair between the model categories $L_{S} \mathrm{C}$ and $L_{\mathbf{L} F S} D$.

We now have the following definition of Quillen equivalence, which is the standard notion of "equivalence" of model category structures.

Definition 2.8. [15, 1.3.12] A Quillen pair is a Quillen equivalence if for all cofibrant $X$ in $\mathcal{C}$ and fibrant $Y$ in $\mathcal{D}$, a map $f: F X \rightarrow Y$ is a weak equivalence in $\mathcal{D}$ if and only if the map $\varphi f: X \rightarrow R Y$ is a weak equivalence in $\mathcal{C}$.

We will use the following proposition to prove that our Quillen pairs are Quillen equivalences. Recall that a functor $F: \mathcal{C} \rightarrow \mathcal{D}$ reflects a property if, for any morphism $f$ of $\mathcal{C}$, whenever $F f$ has the property, then so does $f$.

Proposition 2.9. 15, 1.3.16] Suppose that $(F, R, \varphi)$ is a Quillen pair from $\mathcal{C}$ to $\mathcal{D}$. Then the following statements are equivalent:

(1) $(F, R, \varphi)$ is a Quillen equivalence.

(2) F reflects weak equivalences between cofibrant objects, and for every fibrant $Y$ in $\mathcal{D}$ the map $F(R Y)^{c} \rightarrow Y$ is a weak equivalence.

(3) $R$ reflects weak equivalences between fibrant objects, and for every cofibrant $X$ in $\mathcal{C}$ the map $X \rightarrow R(F X)^{f}$ is a weak equivalence.

\section{Model Category Structures}

In this section, we set up the model category structures that we will need to prove Theorem 1.7.

Because we will need to consider a model structure on the category of simplicial monoids, we now describe a model structure on the category of $\mathcal{T}$-algebras for any algebraic theory $\mathcal{T}$.

Proposition 3.1. [18, II.4], 20, 3.1] Let $\mathcal{T}$ be an algebraic theory and $\mathcal{A l g}{ }^{\mathcal{T}}$ the category of $\mathcal{T}$-algebras. Then there is a cofibrantly generated model category structure on $\mathcal{A l g}^{\mathcal{T}}$ in which the weak equivalences and fibrations are levelwise weak equivalences of simplicial sets and the cofibrations are the maps with the left lifting property with respect to the maps which are fibrations and weak equivalences. 
We would also like to have a model category structure for homotopy $\mathcal{T}$-algebras. However, we will not have a model category structure on the category of homotopy $\mathcal{T}$-algebras itself; the category of homotopy $\mathcal{T}$-algebras does not have all coproducts. We will have a model category structure on the category of all $\mathcal{T}$-diagrams of simplicial sets in which homotopy $\mathcal{T}$-algebras are the fibrant objects. To obtain this structure, we begin by considering the category of all functors $\mathcal{T} \rightarrow \mathcal{S} \mathcal{S} e t s$, which we denote by $\mathcal{S S e t s}^{\mathcal{T}}$.

Theorem 3.2. 12, IX 1.4] There is a model category structure on $\mathcal{S} S e t s^{\mathcal{T}}$ in which the weak equivalences are the levelwise weak equivalences, the fibrations are the levelwise fibrations, and the cofibrations are the maps which have the left lifting property with respect to the maps which are both fibrations and weak equivalences.

The desired model structure can be obtained by localizing the model structure on $\mathcal{S} \mathcal{S e t s}^{\mathcal{T}}$ with respect to a set of maps. We will summarize this localization here; a complete description is given by Badzioch [1, §5].

In an algebraic theory $\mathcal{T}$, consider the functor $\operatorname{Hom}_{\mathcal{T}}\left(T_{k},-\right)$. We then have maps

$$
p_{k}: \coprod_{k} \operatorname{Hom}_{\mathcal{T}}\left(T_{1},-\right) \rightarrow \operatorname{Hom}_{\mathcal{T}}\left(T_{k},-\right)
$$

induced from the projection maps in $\mathcal{T}$. We then localize the model category structure on $\mathcal{S S e t s}^{\mathcal{T}}$ with respect to the set $S=\left\{p_{k} \mid k \geq 0\right\}$. We denote the resulting model category structure $\mathcal{L} \mathcal{S} \mathcal{S} e t s^{\mathcal{T}}$.

Proposition 3.3. [1, 5.5]. The fibrant objects in $\mathcal{L S S e t s}^{\mathcal{T}}$ are the homotopy $\mathcal{T}$ algebras which are fibrant in $\mathcal{S S E}$ st $^{\mathcal{T}}$.

We now have the following result by Badzioch.

Theorem 3.4. 1, 6.4] Given an algebraic theory $\mathcal{T}$, there is a Quillen equivalence of model categories between $\mathcal{A l g}^{\mathcal{T}}$ and $\mathcal{L S S E}_{\text {ets }}{ }^{\mathcal{T}}$.

By applying this Quillen equivalence to the theory of monoids $\mathcal{T}_{M}$, we have reduced the proof of our main theorem to finding a Quillen equivalence between $\mathcal{L S S E}_{\text {Sets }} \mathcal{T}_{M}$ and an appropriate model structure for reduced Segal categories. As with the homotopy $\mathcal{T}$-algebras, we will need to consider the category of reduced Segal precategories and find a model structure in which the fibrant objects are Segal categories.

We consider the general case of the category whose objects are Segal precategories with a fixed set $\mathcal{O}$ in degree zero and whose morphisms are object-preserving on that set. We will prove the existence of a model structure on this category in which the weak equivalences are levelwise weak equivalences of simplicial sets. We give the proof here for any set $\mathcal{O}$, since we will need it for the more general construction in the last section of the paper. Thus, let $\mathcal{S} \mathcal{S} p_{\mathcal{O}}$ denote the category whose objects are simplicial spaces with a fixed set $\mathcal{O}$ in degree zero and whose morphisms are maps of simplicial spaces which are the identity map on $\mathcal{O}$.

This model category structure on $\mathcal{S} \mathcal{S} p_{\mathcal{O}}$ will be analogous to the projective model category structure on simplicial spaces in which the fibrations and weak equivalences are levelwise fibrations and weak equivalences of simplicial sets [12, IX.1.4]. However, this structure must be modified so that the objects defining the generating cofibrations and generating acyclic cofibrations will be in the category $\mathcal{S} \mathcal{S} p_{\mathcal{O}}$. We begin by understanding limits and colimits in $\mathcal{S} \mathcal{S} p_{\mathcal{O}}$. 
Lemma 3.5. $\mathcal{S} S p_{\mathcal{O}}$ has finite limits.

Proof. Suppose that for each object $\alpha$ of an index category $\mathcal{D}, X_{\alpha}$ is a simplicial space with the set $\mathcal{O}$ in degree zero. In the category of all simplicial spaces, we have the limit $\lim _{\mathcal{D}} X_{\alpha}$. However, if $\mathcal{D}$ is not connected, then this limit will not be in the category $\mathcal{S} \mathcal{S} p_{\mathcal{O}}$ since $\lim _{\mathcal{D}}\left(X_{\alpha}\right)_{0}=\mathcal{O}^{\pi_{0}} \mathcal{D}$. However, if diag : $\mathcal{O} \rightarrow \lim _{\mathcal{D}}\left(X_{\alpha}\right)_{0}$ is the diagonal map, we can define the limit in $\mathcal{S} S p_{\mathcal{O}}$, denoted $\lim _{\mathcal{D}}^{\mathcal{O}} X_{\alpha}$, as the pullback in the diagram

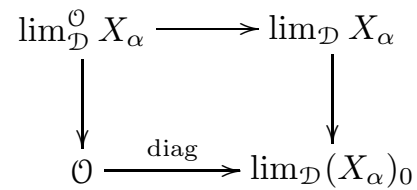

This new object now satisfies the universal property of limits when we require the maps involved to be the identity on degree zero and hence in our category $\mathcal{S} S p_{\mathcal{O}}$.

Lemma 3.6. $\mathcal{S} S p_{\mathcal{O}}$ has finite colimits.

Proof. As with the limits, begin by considering the ordinary colimit in simplicial spaces. Again, let $X_{\alpha}$, indexed by the objects $\alpha$ of $\mathcal{D}$, be objects in $\mathcal{S} \mathcal{S} p_{\mathcal{O}}$. Note that again we have a problem in degree zero if our index category $\mathcal{D}$ has more than one component, since in this case $\operatorname{colim}_{\mathcal{D}}\left(X_{\alpha}\right)_{0}=\coprod_{\pi_{0} \mathcal{D}} \mathcal{O}$. Then if we consider the fold map

$$
\text { fold : } \operatorname{colim}_{\mathcal{D}}\left(X_{\alpha}\right)_{0} \rightarrow \mathcal{O}
$$

we can define the colimit in $\mathcal{S} \mathcal{S} p_{\mathcal{O}}$, denoted $\operatorname{colim}_{D}^{\mathcal{O}} X_{\alpha}$ as the pushout in the diagram

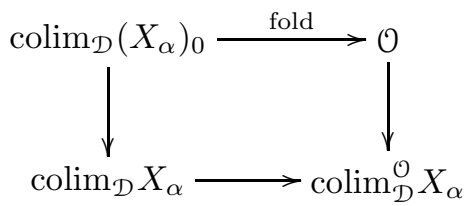

where the left-hand vertical map is the inclusion map. Similarly to the case for limits, this new simplicial space satisfies the universal property for colimits.

Proposition 3.7. There is a model category structure on $\mathcal{S} \mathcal{S} p_{\mathcal{O}}$, which we denote $\mathcal{S} \mathcal{S} p_{\mathcal{O}, f}$, in which the weak equivalences are levelwise weak equivalences of simplicial sets, the fibrations are the levelwise fibrations of simplicial sets, and the cofibrations are the maps with the left lifting property with respect to the maps which are fibrations and weak equivalences.

The first step towards defining this model category structure is finding candidates for the generating cofibrations and generating acyclic cofibrations. Recall that for the projective model category structure on the category $\mathcal{S S E t s}^{\Delta^{o p}}$ of simplicial spaces, in which the weak equivalences and fibrations are levelwise, the generating acyclic cofibrations are of the form

$$
V[m, k] \times \Delta[n]^{t} \rightarrow \Delta[m] \times \Delta[n]^{t}
$$

[12, IV.3.1]. (Recall that by $\Delta[n]^{t}$ we denote the simplicial space which is the constant simplicial set $\Delta[n]_{k}$ in degree $k$.)

The first problem in using these maps for $\mathcal{S} \mathcal{S} p_{\mathcal{O}, f}$ is that $\Delta[n]^{t}$ is not going to be in $\mathcal{S} \mathcal{S} p_{\mathcal{O}}$ for all values of $n$. Instead, we need to define a separate $n$-simplex for 
any $n$-tuple $x_{0}, \ldots, x_{n}$ of objects in $\mathcal{O}$, denoted $\Delta[n]_{x_{0}, \ldots, x_{n}}^{t}$, so that the objects are preserved. Setting $\underline{x}=\left(x_{1}, \ldots, x_{n}\right)$, we write this simplex as $\Delta[n]_{x}^{t}$. (The values of the $x_{i}$ can repeat in a particular $\underline{x}$.) Note that this object $\Delta[n]_{\underline{x}}^{t}$ also needs to have all elements of $\mathcal{O}$ as 0 -simplices, so we add any of these elements that have not already been included in the $x_{i}$ 's, plus their degeneracies in higher degrees.

Now we consider the modified generating acyclic cofibrations

$$
V[m, k] \times \Delta[n]_{\underline{x}}^{t} \rightarrow \Delta[m] \times \Delta[n]_{\underline{x}}^{t} .
$$

However, these objects are still not in $\mathcal{S} S p_{\mathcal{O}, f}$ because the simplicial set in degree zero for each is not discrete. This problem can be fixed by collapsing the copies of $\Delta[m]$ and $V[m, k]$ in degree zero to their respective $x_{i}$ 's. Note that the degeneracies then get collapsed as well. More explicitly, we define the object $\left(R_{m, n, k}\right)_{\underline{x}}$ to be the pushout of the diagram

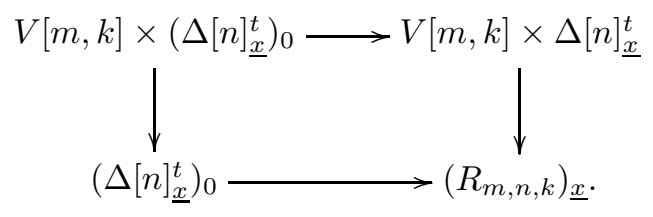

Similarly, we define the object $\left(Q_{m, n}\right)_{\underline{x}}$ to be the pushout of the diagram

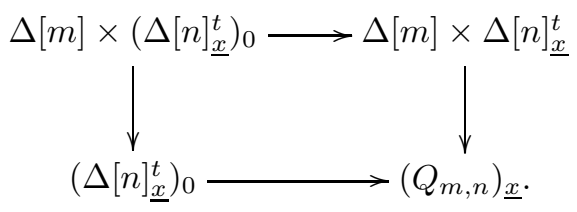

Now we are able to define the set of maps

$$
J_{f}=\left\{\left(R_{m, n, k}\right)_{\underline{x}} \rightarrow\left(Q_{m, n}\right)_{\underline{x}}\right\}
$$

where $m, n \geq 1,0 \leq k \leq m$, and $\underline{x}=\left(x_{0}, \ldots, x_{n}\right) \in \mathcal{O}^{n+1}$. This set $J_{f}$ will be a set of generating acyclic cofibrations for $\mathcal{S} \mathcal{S} p_{\mathcal{O}, f}$.

Similarly, we can define the set

$$
I_{f}=\left\{\left(P_{m, n}\right)_{\underline{x}} \rightarrow\left(Q_{m, n}\right)_{\underline{x}}\right\}
$$

for all $m, n \geq 0$ and $\underline{x} \in \mathcal{O}^{n+1}$, where $\left(P_{m, n}\right)_{\underline{x}}$ is the pushout of the diagram

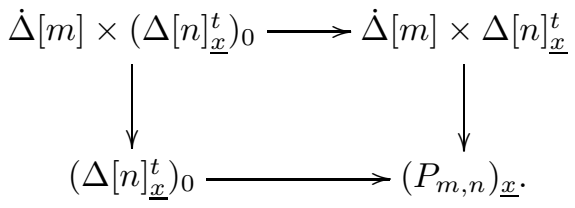

We will show that these maps are a set of generating cofibrations for $\mathcal{S} S p_{\mathcal{O}, f}$.

Proof of Theorem 3.7. Lemmas 3.5 and 3.6 show that our category has finite limits and colimits. The two-out-of-three property and the retract axiom for weak equivalences follow as usual; see for example [11, 8.10]. It now suffices to check the conditions of Theorem 2.2 To prove statement (1), notice that the maps in the sets $I_{f}$ and $J_{f}$ are modified versions of the generating cofibrations in the projective model category structure on simplicial spaces, which permit the small object argument [13, 10.5.15]. Hence the ones in $I_{f}$ and $J_{f}$ do also. 
Notice that the $I_{f}$-injectives are precisely the levelwise acyclic fibrations, and that the $J_{f}$-injectives are precisely the levelwise fibrations. Thus, we have satisfied conditions (3) and (4)(ii).

Now notice that the $I_{f}$-cofibrations are precisely the cofibrations, by our definition of cofibration. Furthermore, the $J_{f}$-cofibrations are the maps with the left lifting property with respect to the fibrations. In particular, they are the acyclic cofibrations in the projective model category structure on simplicial spaces which are between Segal precategories and leave the degree zero space fixed. Since the cofibrations can be described analogously, condition (2) holds, completing the proof.

We now want to localize this model category so that the fibrant objects are Segal categories with the set $\mathcal{O}$ in degree zero. If we can find an appropriate map to localize with respect to, then the desired model category structure will follow from Theorem 2.5.

We first consider the map $\varphi$ used by Rezk [19, §4] to localize simplicial spaces to obtain more general Segal spaces, then modify it so that the objects are in $\mathcal{S} S p_{\mathcal{O}, f}$.

Rezk defines a map $\alpha^{i}:[1] \rightarrow[k]$ in $\boldsymbol{\Delta}$ such that $0 \mapsto i$ and $1 \mapsto i+1$ for each $0 \leq i \leq k-1$. Then for each $k$ he defines the object

$$
G(k)^{t}=\bigcup_{i=0}^{k-1} \alpha^{i} \Delta[1]^{t}
$$

and the inclusion map $\varphi^{k}: G(n)^{t} \rightarrow \Delta[k]^{t}$. His localization is with respect to the coproduct of inclusion maps

$$
\varphi=\coprod_{k \geq 0}\left(G(k)^{t} \rightarrow \Delta[k]^{t}\right) .
$$

However, in our case, the objects $G(k)^{t}$ and $\Delta[k]^{t}$ are not in the category $\mathcal{S} \mathcal{S} p_{\mathcal{O}}$. As before, we can replace $\Delta[k]^{t}$ with the objects $\Delta[k]_{\underline{x}}^{t}$, where $\underline{x}=\left(x_{0}, \ldots, x_{k}\right)$. Then, define

$$
G(k)_{\underline{x}}^{t}=\bigcup_{i=0}^{k-1} \alpha^{i} \Delta[1]_{x_{i}, x_{i+1}}^{t} .
$$

Now, we need to take coproducts not only over all values of $k$, but also over all $k$-tuples of vertices.

We first define for each $k \geq 0$ the map

$$
\varphi^{k}=\coprod_{\underline{x} \in \mathcal{O}^{k+1}}\left(G(k)_{\underline{x}}^{t} \rightarrow \Delta[k]_{\underline{x}}^{t}\right) .
$$

Then the map $\varphi$ looks like

$$
\varphi=\coprod_{k \geq 0}\left(\varphi^{k}: \coprod_{\underline{x} \in \mathcal{O}^{k+1}}\left(G(k)_{\underline{x}}^{t} \rightarrow \Delta[k]_{\underline{x}}^{t}\right)\right) .
$$

When the set $\mathcal{O}$ is not clear from the context, we will write $\varphi_{\mathcal{O}}$ to specify that we are in $\mathcal{S} S p_{\mathcal{O}}$.

For any simplicial space $X$, there is a weak equivalence of simplicial sets

$$
\operatorname{Map}^{h}\left(\coprod_{\underline{x} \in \mathcal{O}^{k+1}} G(k)_{\underline{x}}^{t}, X\right) \rightarrow \underbrace{X_{1} \times_{X_{0}}^{h} \cdots \times_{X_{0}}^{h} X_{1}}_{k}
$$


where the right-hand side is the homotopy limit of the diagram

$$
X_{1} \stackrel{d_{0}}{\longrightarrow} X_{0} \stackrel{d_{1}}{\longleftarrow} X_{1} \stackrel{d_{0}}{\longrightarrow} \cdots \stackrel{d_{0}}{\longrightarrow} X_{0} \stackrel{d_{1}}{\longleftarrow} X_{1}
$$

with $k$ copies of $X_{1}$. However, in this case, since $X_{0}$ is discrete we can take the limit

$$
\underbrace{X_{1} \times_{X_{0}} \cdots \times_{X_{0}} X_{1}}_{k}
$$

on the right hand side.

Then for any simplicial space $X$ there is a map

$$
\varphi_{k}=\operatorname{Map}^{h}\left(\varphi^{k}, X\right): \operatorname{Map}^{h}\left(\coprod_{\underline{x}} \Delta[k]_{\underline{x}}^{t}, X\right) \rightarrow \operatorname{Map}^{h}\left(\coprod_{\underline{x}} G(k)_{\underline{x}}^{t}, X\right) .
$$

More simply written, this map is

$$
\varphi_{k}: X_{k} \rightarrow \underbrace{X_{1} \times_{X_{0}} \cdots \times_{X_{0}} X_{1}}_{k}
$$

and is often called a Segal map.

Localizing with respect to this map $\varphi$, then, will result in a model category structure in which the local objects are those objects $X$ of $\mathcal{S} S p_{\mathcal{O}, f}$ for which each Segal map $X_{k} \rightarrow \underbrace{X_{1} \times_{\mathcal{O}} \cdots \times_{\mathcal{O}} X_{1}}_{k}$ is a weak equivalence of simplicial sets.

Proposition 3.8. Localizing the model category structure on $\mathcal{S} \mathcal{S} p_{\mathcal{O}, f}$ with respect to the map $\varphi_{\mathcal{O}}$ results in a model category structure $\mathcal{L} \mathcal{S} \mathcal{S}_{p_{\mathcal{O}, f}}$ on simplicial spaces with a fixed set $\mathcal{O}$ in degree zero in which the weak equivalences are the $\varphi_{\mathcal{O}}$-local equivalences, the cofibrations are those of $\mathcal{S} \mathcal{S} p_{\mathcal{O}, f}$, and the fibrations are the maps with the right lifting property with respect to the cofibrations which are $\varphi_{\mathcal{O}}$-local equivalences. Furthermore, the fibrant objects are the Segal categories which are fibrant in $\mathcal{S} S p_{\mathcal{O}, f}$.

Proof. The proof follows from Theorem 2.5 and the above argument.

For making some of our calculations, we will find it convenient to work in a model category structure $\mathcal{S} S p_{\mathcal{O}, c}$ in which the weak equivalences are again given by levelwise weak equivalences of simplicial sets, but in which the cofibrations, rather than the fibrations, are levelwise.

Theorem 3.9. There is a model category structure $\mathcal{S} \mathcal{S} p_{\mathcal{O}, c}$ on the category of Segal precategories with a fixed set $\mathcal{O}$ in degree zero in which the weak equivalences and cofibrations are levelwise, and in which the fibrations are the maps with the right lifting property with respect to the acyclic cofibrations.

To define sets $I_{c}$ and $J_{c}$ which will be our candidates for generating cofibrations and generating acyclic cofibrations, respectively, we first recall the generating cofibrations and acyclic cofibrations in the Reedy (or injective) model category structure on simplicial spaces, in which the weak equivalences and cofibrations are levelwise. The generating cofibrations are the maps

$$
\dot{\Delta}[m] \times \Delta[n]^{t} \cup \Delta[m] \times \dot{\Delta}[n]^{t} \rightarrow \Delta[m] \times \Delta[n]^{t}
$$

for all $m, n \geq 0$, and similarly the generating acyclic cofibrations are the maps

$$
V[m, k] \times \Delta[n]^{t} \cup \Delta[m] \times \dot{\Delta}[n]^{t} \rightarrow \Delta[m] \times \Delta[n]^{t}
$$


for all $n \geq 0, m \geq 1$, and $0 \leq k \leq m$ [19, 2.4].

To modify these maps, we begin by considering the category $\mathcal{S} e \mathcal{C} a t$ of all Segal precategories and the inclusion functor $\mathcal{S} e \mathrm{C} a t \rightarrow \mathcal{S S e t}^{\boldsymbol{\Delta}^{o p}}$. This functor has a left adjoint which we call the reduction functor. Given a simplicial space $X$, we denote its reduction by $(X)_{r}$. Reducing $X$ essentially amounts to collapsing the space $X_{0}$ to its set of components and making the appropriate changes to degeneracies in higher degrees. So, we start by reducing the objects defining the Reedy generating cofibrations and generating acyclic cofibrations to obtain maps of the form

$$
\left(\dot{\Delta}[m] \times \Delta[n]^{t} \cup \Delta[m] \times \dot{\Delta}[n]^{t}\right)_{r} \rightarrow(\Delta[m] \times \Delta[n])_{r}
$$

and

$$
\left(V[m, k] \times \Delta[n]^{t} \cup \Delta[m] \times \dot{\Delta}[n]^{t}\right)_{r} \rightarrow\left(\Delta[m] \times \Delta[n]^{t}\right)_{r}
$$

Then, in order to have our maps in $\mathcal{S} S p_{\mathcal{O}}$, we define a separate such map for each choice of vertices $\underline{x}$ in degree zero and adding in the remaining points of $\mathcal{O}$ if necessary. As above, we use $\Delta[n]_{\underline{x}}^{t}$ to denote the object $\Delta[n]^{t}$ with the $(n+1)$-tuple $\underline{x}$ of vertices. We then define sets

$$
I_{c}=\left\{\left(\dot{\Delta}[m] \times \Delta[n]_{\underline{x}}^{t} \cup \Delta[m] \times \dot{\Delta}[n]_{\underline{x}}^{t}\right)_{r} \rightarrow\left(\Delta[m] \times \Delta[n]_{\underline{x}}^{t}\right)_{r}\right\}
$$

for all $m \geq 0$ and $n \geq 1$, and

$$
J_{c}=\left\{\left(V[m, k] \times \Delta[n]_{\underline{x}}^{t} \cup \Delta[m] \times \dot{\Delta}[n]_{\underline{x}}^{t}\right)_{r} \rightarrow\left(\Delta[m] \times \Delta[n]_{\underline{x}}^{t}\right)_{r}\right\}
$$

for all $m \geq 1, n \geq 1$, and $0 \leq k \leq m$, where the notation $(-)_{x}$ indicates the vertices.

Given these maps, we are now able to prove the existence of the model category structure $\mathcal{S} S p_{\mathcal{O}, c}$.

Proof of Theorem 3.9. The proofs that $\mathcal{S} S p_{\mathcal{O}, c}$ has finite limits and colimits and satisfies the two out of three and retract axioms, as well as condition (1), are the same as for $\mathcal{S} \mathcal{S} p_{\mathcal{O}, f}$.

From the definitions of $I_{c}$ and $J_{c}$, it follows that the $I_{c}$-injectives are the maps of Segal precategories with $\mathcal{O}$ in degree zero which are Reedy fibrations and that the $J_{c}$-injectives are the maps of Segal precategories with $\mathcal{O}$ in degree zero which are Reedy acyclic fibrations. Furthermore, it follows from these facts that the $I_{c^{-}}$ cofibrations are precisely the cofibrations and that the $J_{c}$-cofibrations are precisely the acyclic cofibrations. These observations imply that the conditions of Theorem 2.2 are satisfied.

We can then localize $\mathcal{S} S p_{\mathcal{O}, c}$ with respect to the map $\varphi$ to obtain a model category structure which we denote $\mathcal{L} \mathcal{S} S p_{\mathcal{O}, c}$.

At first glance, one might wonder if the weak equivalences in $\mathcal{L} \mathcal{S} S p_{\mathcal{O}, f}$ and $\mathcal{L} \mathcal{S} S p_{\mathcal{O}, c}$ are actually the same, since each of these model structures is obtained via localization of a different model structure on Segal precategories. However, since the weak equivalences before localization are the same in each case, this localization is independent of the underlying model structure [5] $\$ 7]$. Therefore, the weak equivalences are actually the same in these two localized structures. (Notice that here we need to use the more general notion of homotopy function complex in a category with specified weak equivalences, rather than in a simplicial model category, since we are working in two different model categories at once.)

We then have the following result. 
Proposition 3.10. The adjoint pair given by the identity functor induces a Quillen equivalence of model categories

$$
\mathcal{L S S} p_{\mathcal{O}, f} \rightleftarrows \mathcal{L S S} p_{\mathcal{O}, c} .
$$

Proof. Since the cofibrations in $\mathcal{L} \mathcal{S} \mathcal{S} p_{\mathcal{O}, f}$ are monomorphisms, the identity functor

$$
\mathcal{L} \mathcal{S S} p_{\mathcal{O}, f} \rightarrow \mathcal{L S S S} p_{\mathcal{O}, c}
$$

preserves both cofibrations and acyclic cofibrations, so this adjoint pair is a Quillen pair. It remains to show that for any cofibrant $X$ in $\mathcal{L} \mathcal{S} S p_{\mathcal{O}, f}$ and fibrant $Y$ in $\mathcal{L} \mathcal{S} S p_{\mathcal{O}, c}$, the map $F X \rightarrow Y$ is a weak equivalence if and only of the map $X \rightarrow R Y$ is a weak equivalence. However, this fact follows since the weak equivalences are the same in each category.

Before proceeding to the main proof, we need one more model structure. Let * denote the set with one element. The objects of $\mathcal{L} \mathcal{S} S p_{*, f}$ have a single point in degree zero, whereas the objects in $\mathcal{L S S E} s^{\mathcal{T}_{M}}$ have an arbitrary simplicial set in degree zero. To simplify matters, we define a model structure analogous to $\mathcal{L S S E t s}^{\mathcal{T}_{M}}$ but on the category of functors $\mathcal{T}_{M} \rightarrow$ Sets which send $T_{0}$ to $\Delta[0]$.

Proposition 3.11. Consider the category SSets $s_{*}^{\mathcal{T}_{M}}$ of functors $\mathcal{T}_{M} \rightarrow$ SSets such that the image of $T_{0}$ is $\Delta[0]$. There is a model category structure on $\mathcal{S S S e t}_{*}^{\mathcal{T}_{M}}$ in which the weak equivalences and fibrations are defined levelwise and the cofibrations are the maps with the left lifting property with respect to the acyclic fibrations.

Proof. Limits and colimits exist in $\mathcal{S} \mathcal{S} e t s_{*}^{\mathcal{T}_{M}}$ by analogous arguments to the ones given in Lemmas 3.5 and 3.6 By taking sets of generating cofibrations and generating acyclic cofibrations for $\mathcal{S S e t s}^{\mathcal{T}_{M}}$ and modifying them in the same way as we did for $\mathcal{S} \mathcal{S}_{*, f}$, we can obtain generating sets for $\mathcal{S} \mathcal{S} e t s_{*}^{\mathcal{T}_{M}}$. The proof follows just as the proof of Theorem 3.7

Now, to obtain a localized model category $\mathcal{L} \mathcal{S} \mathcal{S} e t s_{*}^{\mathcal{T}_{M}}$, we need to modify the maps

$$
p_{k}: \coprod_{k} \operatorname{Hom}_{\mathcal{T}}\left(T_{1},-\right) \rightarrow \operatorname{Hom}_{\mathcal{T}}\left(T_{k},-\right)
$$

that we used to obtain $\mathcal{L} \mathcal{S} \mathcal{S} e t s^{\mathcal{T}_{M}}$ from $\mathcal{S S E}$ ets ${ }^{\mathcal{T}_{M}}$. Since $\operatorname{Hom}_{\mathcal{T}}\left(T_{k},-\right)_{0} \cong \Delta[0]$ for all $k \geq 0$, the only change we need to make to these maps is to take the coproduct $\coprod_{k} \operatorname{Hom}_{\mathcal{T}}\left(T_{1},-\right)$ in the category $\mathcal{S S e t s}_{*}^{\mathcal{T}_{M}}$ (as in Lemma 3.6). We then localize $\mathcal{S S e t s}_{*}^{\mathcal{T}_{M}}$ with respect to the set of all such maps to obtain a model structure $\mathcal{L} \mathcal{S S e t}_{*}^{\mathcal{T}_{M}}$.

Since a fibrant and cofibrant object $X$ in $\mathcal{L S S E}$ st $^{\mathcal{T}_{M}}$ has $X_{0}$ weakly equivalent to $\Delta[0]$, it is not too surprising that we have the following result:

Proposition 3.12. There is a Quillen equivalence of model categories

$$
\mathcal{L S S e t s}_{*}^{\mathcal{T}_{M}} \rightleftarrows \mathcal{L} \mathcal{S S e t s}^{\mathcal{T}_{M}} .
$$

Proof. There is an inclusion map $I: \mathcal{L S S E} s_{*}^{\mathcal{T}_{M}} \rightarrow \mathcal{L S S E} \boldsymbol{\mathcal { S }}^{\mathcal{T}_{M}}$ whose right adjoint $C$ collapses the space in degree zero to a point. Since the cofibrations of $\mathcal{L S S E} s_{*}^{\mathcal{T}_{M}}$ are also cofibrations in $\mathcal{L S S E}^{\mathcal{T}_{M}}$ and the weak equivalences are defined in the same way in each model structure, $I$ preserves cofibrations and acyclic cofibrations. Hence, this adjoint pair is a Quillen pair. 
By the same argument, $I$ also reflects weak equivalences between cofibrant objects. To prove that we have a Quillen equivalence, it remains to show that for any fibrant object $Y$ in $\mathcal{L S S E}_{\text {ets }}{ }^{\mathcal{T}_{M}}$, the map $I(C Y)^{c} \rightarrow Y$ is a weak equivalence. However, $Y$ is a homotopy $\mathcal{T}_{M}$-algebra and hence $Y_{0}$ is weakly equivalent to $\Delta[0]$. Therefore, up to homotopy $Y$ is not changed by applying the functor $C$, and it follows that this maps is in fact a weak equivalence.

\section{Proof of Theorem 1.7}

For the rest of this section, we will set $\mathcal{O}=*$, the one-element set. In the previous section, we used Theorem 3.4 to see that the model category structure on $\mathcal{A l g}{ }^{\mathcal{T}_{M}}$, which is equivalent to the category of simplicial monoids, is Quillen equivalent to $\mathcal{L S S E t s}^{\mathcal{T}_{M}}$. This model category is in turn Quillen equivalent to $\mathcal{L S S E t}_{*}^{\mathcal{T}_{M}}$ by Proposition 3.12 So, to prove Theorem 1.7 it suffices to show that there is a Quillen equivalence between the model categories $\mathcal{L} \mathcal{S} \mathcal{S} p_{*, f}$ and $\mathcal{L} \mathcal{S} \mathcal{S} e t s_{*}^{\mathcal{T}_{M}}$.

Let $L_{1}$ be the functorial fibrant replacement functor (or localization) for $\mathcal{L} \mathcal{S} \mathcal{S} p_{*}$ given by taking a colimit of pushouts along the generating acyclic cofibrations, and let $L_{2}$ be the analogous fibrant replacement functor for $\mathcal{L} \mathcal{S} \mathcal{S} e t s_{*}^{\mathcal{T}_{M}}$.

We would like to know what the localization $L_{1}$ does to an $n$-simplex $\Delta[n]_{*}^{t}$. Note that we will use this notation in the sense of the previous section: we are specifying that we are working in the category of reduced Segal precategories, namely in the case where $\mathcal{O}=*$. To make the calculations about our localizations, we will use the model structure $\mathcal{L} \mathcal{S} S p_{*, c}$, since in this case all objects are cofibrant and in particular all monomorphisms are cofibrations.

Lemma 4.1. Let $L$ be a localization functor on a model category $\mathcal{M}$. Given a small diagram of objects $X_{\alpha}$ of $\mathcal{M}$,

$$
L\left(\operatorname{hocolim} X_{\alpha}\right) \simeq \operatorname{Lhocolim} L\left(X_{\alpha}\right) .
$$

Proof. It suffices to show that for any local object $Y$, there is a weak equivalence of simplicial sets

$$
\operatorname{Map}^{h}\left(L\left(\operatorname{hocolim}_{\alpha} L X_{\alpha}\right), Y\right) \simeq \operatorname{Map}^{h}\left(\operatorname{Lhocolim}_{\alpha} X_{\alpha}, Y\right) .
$$

This fact follows from the following series of weak equivalences:

$$
\begin{aligned}
\operatorname{Map}^{h}\left(\operatorname{Lhocolim}_{\alpha} L X_{\alpha}, Y\right) & \simeq \operatorname{Map}^{h}\left(\operatorname{hocolim}_{\alpha} L X_{\alpha}, Y\right) \\
& \simeq \operatorname{holim}_{\alpha} \operatorname{Map}^{h}\left(L X_{\alpha}, Y\right) \\
& \simeq \operatorname{holim}_{\alpha} \operatorname{Map}^{h}\left(X_{\alpha}, Y\right) \\
& \simeq \operatorname{Map}^{h}\left(\operatorname{hocolim}_{\alpha} X_{\alpha}, Y\right) \\
& \simeq \operatorname{Map}^{h}\left(\operatorname{Lhocolim}_{\alpha} X_{\alpha}, Y\right) .
\end{aligned}
$$

We now consider the simplicial space nerve $\left(T_{n}\right)^{t}$, which is the nerve of the free monoid on $n$ generators, considered as a transposed simplicial space.

Proposition 4.2. Let $F_{n}$ denote the free monoid on n generators. Then in $\mathcal{L} \mathcal{S} S p_{*, c}$, $L_{1} \Delta[n]_{*}^{t}$ is weakly equivalent to nerve $\left(T_{n}\right)^{t}$ for each $n \geq 0$. 
Proof. Note that when $n=0, \Delta[0]_{*}^{t}$ is isomorphic to nerve $\left(T_{0}\right)^{t}$, which is already a Segal category.

Now consider the case where $n=1$. We want to show that the map $\Delta[1]_{*}^{t} \rightarrow$ nerve $\left(T_{1}\right)^{t}$ obtained by localizing with respect to the map $\varphi_{*}$ is a weak equivalence in $\mathcal{L} \mathcal{S} S p_{*, c}$. In order to do this, we define a filtration of nerve $\left(T_{1}\right)^{t}$. Let the $k$-th stage of the filtration be

$$
\Psi_{k}\left(\text { nerve }\left(T_{1}\right)^{t}\right)_{j}=\left\{\left(x^{n_{1}}|\cdots| x^{n_{j}}\right) \mid \sum n_{j} \leq k\right\} .
$$

Thus we have

$$
\Delta[1]_{*}^{t}=\Psi_{1} \subseteq \Psi_{2} \subseteq \cdots \subseteq \Psi_{k} \subseteq \cdots \operatorname{nerve}\left(T_{1}\right)^{t} .
$$

Note that the fact that $\Delta[1]_{*}^{t}=\Psi_{1}$ can be observed from looking at the bar construction notation of each as a simplicial set (which we then view as a simplicial space by transposing it). Each has one nondegenerate 1-simplex which we denote by $x$.

Note that $\Psi_{1}$ has no nondegenerate 2-simplices. However, we want to define the "composite" of $x$ with itself, a 1-simplex which we denote by $x^{2}$, and add a nondegenerate 2-simplex $[x, x]$ whose boundary consists of the 1-simplices $x, x$ and $x^{2}$. More formally, take the object $G(2)_{*}^{t}$ as defined in the last section (which lives in $\Psi_{2}$ ) and add another 1-simplex $x^{2}$ and the 2-simplex $[x, x]$ in $\Psi_{2}$. We can describe this passing from $\Psi_{1}$ to $\Psi_{2}$ by the following pushout diagram:

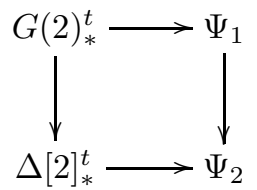

Since we are working in $\mathcal{S} S p_{*, c}$, the left-hand vertical map is an acyclic cofibration, and therefore $\Psi_{1} \rightarrow \Psi_{2}$ is an acyclic cofibration also [1] 3.14].

Similarly, to obtain $\Psi_{3}$ we will add an extra 1-simplex, denoted $x^{3}$, in order to add a 3 -simplex $[x, x, x]$. However, when taking the pushout, we do not want to start with $G(3)_{*}$, since we have already added two of the new 1-simplices when we localized to obtain $\Psi_{2}$. So, we define $\left(\Delta[3]_{*}^{t}\right)_{\Psi_{2}}$ to be the piece of $\Delta[3]_{*}^{t}$ that we already have in $\Psi_{2}$. Then our pushout looks like:

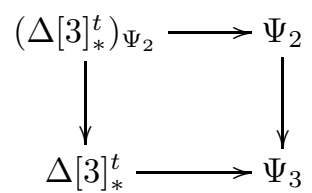

The map $\left(\Delta[3]_{*}^{t}\right)_{\Psi_{2}} \rightarrow \Delta[3]_{*}^{t}$ is a weak equivalence in $\mathcal{L} \mathcal{S} \mathcal{S} p_{*, c}$ as follows. We have maps

$$
G(3)_{*}^{t} \stackrel{\alpha}{\longrightarrow}\left(\Delta[3]_{*}^{t}\right)_{\Psi_{2}} \stackrel{\beta}{\longrightarrow} \Delta[3]_{*}^{t} .
$$

Taking the function complex $\operatorname{Map}(-, X)$ for any local $X$ for any of the three above spaces yields $X_{1} \times X_{1} \times X_{1} \simeq X_{3}$. The map $\alpha$ is a weak equivalence since it is just a patching together of two localizations coming from the map $G(2)_{*}^{t} \rightarrow \Delta[2]_{*}^{t}$, which is a weak equivalence since it is one of the maps with respect to which we are localizing. The composite map $\beta \alpha$ is also a weak equivalence for the same reason. Thus, $\beta$ is also a weak equivalence by the two-out-of-three property. Again, since 
$\left(\Delta[3]_{*}^{t}\right)_{\Psi_{2}} \rightarrow \Delta[3]_{*}^{t}$ is an acyclic cofibration in $\mathcal{L} \mathcal{S} \mathcal{S} p_{*, c}$, the map $\Psi_{2} \rightarrow \Psi_{3}$ is an acyclic cofibration also.

For greater values of $i$, define $\left(\Delta[i+1]_{*}^{t}\right)_{\Psi_{i}}$ to be the piece of $\Delta[i+1]_{*}^{t}$ that we already have from previous steps of the filtration. Note that it is always two copies of $\Delta[i]_{*}^{t}$ attached along a copy of $\Delta[i-1]_{*}^{t}$, so the same argument as for $i=2$ shows that the map $\left(\Delta[i+1]_{*}^{t}\right)_{\Psi_{i}} \rightarrow \Delta[i+1]_{*}^{t}$ is a weak equivalence. Hence, for each $i$ we obtain $\Psi_{i+1}$ via the pushout diagram

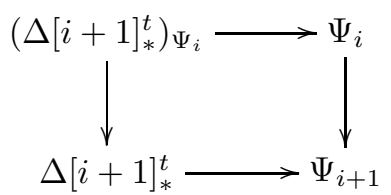

Now that we have defined each stage of our filtration, using the bar construction notation shows how to map our new local object to nerve $\left(T_{1}\right)^{t}$. For example, $\left[x, x, x^{2}\right] \mapsto\left(x, x, x^{2}\right) \in T_{1} \times T_{1} \times T_{1}$.

Using Lemma 4.1 we have that

$$
\begin{aligned}
\operatorname{nerve}\left(T_{1}\right)^{t} & \simeq L_{1}\left(\operatorname{nerve}\left(T_{1}\right)^{t}\right) \\
& \simeq L_{1}\left(\operatorname{hocolim}\left(\Psi_{i}\right)\right) \\
& \simeq L_{1}\left(\operatorname{hocolim} L_{1}\left(\Psi_{i}\right)\right) \\
& \simeq L_{1}\left(\operatorname{hocolim} L_{1}\left(\Psi_{1}\right)\right) \\
& \simeq L_{1} L_{1}\left(\Psi_{1}\right) \\
& \simeq L_{1}\left(\Psi_{1}\right) \\
& \simeq L_{1}\left(\Delta[1]_{*}^{t}\right)
\end{aligned}
$$

Now, for $n=2$ (i.e. starting with $\Delta[2]_{*}^{t}$ ), we have three 1-simplices, which we will call $x, y$, and $x y$, and one nondegenerate 2 -simplex $[x, y]$. Because we now have two variables, we need to define the filtration slightly differently as $\Psi_{i}=$ $\left\{\left[w_{1}, \ldots, w_{k}\right] \mid l\left(w_{1} \ldots w_{k}\right) \leq i\right\}$ where the $w_{j}$ 's are words in $x$ and $y$ and $l$ denotes the length of a given word. Note that by beginning with $\Psi_{1}$ we start with fewer simplices than those of the 2-simplex we are considering, but by passing to $\Psi_{2}$ we obtain the $x y$ and $[x, y]$ as well as additional nondegenerate simplices. In fact, we are actually starting the filtration with $\Psi_{1}=G(2)_{*}^{t}$. The localizations proceed as in the case where $n=1$, enabling us to map to nerve $\left(T_{2}\right)^{t}$.

For $n \geq 3$, the same argument works as for $n=2$, with the filtrations being defined by the lengths of words in $n$ letters. The resulting object is a reduced Segal category weakly equivalent to $\Delta[n]_{*}^{t}$. Hence, we have that for any $n, L_{1} \Delta[n]_{*}^{t}$ is weakly equivalent to nerve $\left(T_{n}\right)^{t}$.

We now define a map $J: \boldsymbol{\Delta}^{o p} \rightarrow \mathcal{T}_{M}$ induced by the nerve construction on a monoid $M$. We will actually define the map $J^{o p}: \Delta \rightarrow \mathcal{T}_{M}^{o p}$. (Note that $\mathcal{T}_{M}^{o p}$ is just the full subcategory of the category of monoids whose objects are the isomorphism classes of free monoids.)

For an object $[n]$ of $\boldsymbol{\Delta}$, define $J^{o p}([n])=T_{n}$ where $T_{n}$ denotes the free monoid on $n$ generators, say $x_{1}, \ldots, x_{n}$. In particular, $J^{o p}([0])=*$, the trivial monoid. There are coface and codegeneracy maps induced from the nerve construction on $M$ as follows. 
Taking the nerve of a simplicial monoid $M$ and regarding it as a constant simplicial space results in a simplicial space which at level $k$ looks like

$$
\operatorname{nerve}(M)_{k}=M^{k}=\operatorname{Hom}_{\mathcal{M} \text { on }}\left(T_{k}, M\right) \text {. }
$$

By Yoneda's Lemma, the face operators

$$
d_{i}: \text { nerve }(-)_{k} \rightarrow \operatorname{nerve}(-)_{k-1}
$$

are induced by monoid maps $T_{k-1} \rightarrow T_{k}$, and similarly for the degeneracy maps

$$
s_{i}: \text { nerve }(-)_{k} \rightarrow \text { nerve }(-)_{k+1} \text {. }
$$

Thus the simplicial diagram nerve( $(-)$ of representable functors $\operatorname{Hom}\left(T_{k},-\right)$ gives rise to a cosimplicial diagram of representing objects $T_{k}$. In particular, the coface maps are defined by:

$$
d^{i}\left(x_{k}\right)= \begin{cases}x_{k} & i<k \\ x_{k} x_{k+1} & i=k \\ x_{k+1} & i>k\end{cases}
$$

and the codegeneracy maps are defined analogously. To obtain a simplicial diagram of free monoids, we simply reverse the direction of the arrows to obtain a functor $J: \boldsymbol{\Delta}^{o p} \rightarrow \mathcal{T}_{M}$. This map induces a map $J^{*}: \mathcal{S S}$ ets ${ }^{\mathcal{T}_{M}} \rightarrow \mathcal{S S E t s}^{\boldsymbol{\Delta}^{o p}}$ which can be restricted to a map $J^{*}: \mathcal{S} \mathcal{S} e t s_{*}^{\mathcal{T}_{M}} \rightarrow \mathcal{S} \mathcal{S} p_{*}$.

We now state two definitions in the following general context. Let $p: \mathcal{C} \rightarrow \mathcal{D}$, $G: \mathcal{C} \rightarrow$ SSets, and $T: \mathcal{D} \rightarrow$ SSets be functors.

Definition 4.3. If $d$ is an object of $\mathcal{D}$, then the over category or category of objects over $d$, denoted $(p \downarrow d)$, is the category whose objects are pairs $(c, f)$ where $c$ is an object of $\mathcal{C}$ and $f: p(c) \rightarrow d$ is a morphism in $\mathcal{D}$. If $c^{\prime}$ is another object of $\mathcal{C}$, a morphism in the over category is given by a map $p(c) \rightarrow p\left(c^{\prime}\right)$ inducing a commutative triangle

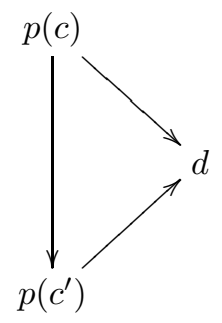

Definition 4.4. 13, 11.8.1] Let $p, c$, and $G$ be defined as above, and let $f: p(c) \rightarrow d$ be an object in $(p \downarrow d)$. The left Kan extension over $p$ is a functor $p_{*} G: \mathcal{D} \rightarrow \mathcal{S}$ Sets defined by

$$
\left(p_{*} G\right)(d)=\operatorname{colim}_{(p \downarrow d)}((c, f) \mapsto G(c)) .
$$

Note. Ordinarily, we would have to take a homotopy left Kan extension, where we replace the colimit in the definition with a homotopy colimit, to make sure that our calculations were homotopy invariant. However, since we are making our calculations in $\mathcal{L} \mathcal{S S} p_{*, c}$ where all objects are cofibrant, and since left Kan extensions agree with homotopy left Kan extensions on cofibrant objects [10, 3.7], the left Kan extension is sufficient. 
Proposition 4.5. [13, 11.9.3] The functor $\mathcal{S S E t s}^{\mathcal{C}} \rightarrow \mathcal{S S E}$ ets ${ }^{\mathcal{D}}$ given by sending $G$ the left Kan extension $p_{*} G$ is left adjoint to the functor $\mathcal{S} S$ ets ${ }^{\mathcal{D}} \rightarrow \mathcal{S S E}^{\mathrm{S} e t}{ }^{\mathcal{C}}$ given by composition with $p$.

In our specific case, we define $J_{*}: \mathcal{S} \mathcal{S} p_{*} \rightarrow \mathcal{S} \mathcal{S} e t s_{*}^{\mathcal{T}_{M}}$ to be the left Kan extension over $J$ which is, by definition, the left adjoint to $J^{*}$. Note that even if $G$ is a reduced Segal category, $J_{*}(G)$ is not necessarily local in $\mathcal{L S S E}_{e} s_{*}^{\mathcal{T}_{M}}$. To obtain a $\mathcal{T}_{M}$-algebra, we must apply the localization functor $L_{2}$.

Define $M[k]$ to be the $\mathcal{T}_{M}$-space with $n$ copies of $T_{k}$ at level $n$. Let $G$ be the reduced Segal category nerve $\left(T_{k}\right)^{t}$.

Lemma 4.6. In $\mathcal{L S S e t s}_{*}^{\mathcal{T}_{M}}, L_{2} J_{*}(G)$ is weakly equivalent to $M[k]$.

Proof. It suffices to show that for any local object $X$ in $\mathcal{L S S E}_{e} s_{*}^{\mathcal{T}_{M}}$,

$$
\operatorname{Map}_{\mathcal{L S S e t s}_{*}^{\tau_{M}}}^{h}\left(L_{2} J_{*}^{h} G, X\right) \simeq X\left(T_{k}\right)
$$

since $M[k]$ is precisely $X\left(T_{k}\right)$. This fact can be shown in the following argument:

$$
\begin{aligned}
& \operatorname{Map}_{\mathcal{L} \operatorname{SSets}_{*}^{\mathcal{T}_{M}}}^{h}\left(L_{2} J_{*} G, X\right) \simeq \operatorname{Map}_{\mathcal{L} \operatorname{SSets}_{*}^{\mathcal{T}_{M}}}^{h}\left(J_{*} G, X\right) \\
& \simeq \operatorname{Map}_{\mathcal{L} S \mathcal{S} p_{*, c}}^{h}\left(G, J^{*} X\right) \\
& \simeq \operatorname{Map}_{\mathcal{L} S \delta p_{*, c}}^{h}\left(L_{1} \Delta[k]_{*}^{t}, J^{*} X\right) \\
& \simeq \operatorname{Map}_{\mathcal{L} \mathcal{S} p_{*, c}}^{h}\left(\Delta[k]_{*}^{t}, J^{*} X\right) \\
& \simeq J^{*} X[k] \\
& \simeq X\left(T_{k}\right) \text {. }
\end{aligned}
$$

Note that this lemma, combined with Lemma 4.2 shows that $L_{2} J_{*}\left(\Delta[k]_{*}^{t}\right) \simeq$ $M[k]$. (Actually, this fact is true even without localizing the left-hand side because $\Delta[k]_{*}^{t}$ is free.)

Proposition 4.7. For any object $X$ in $\mathcal{S} S p_{*, c}$, we have that $L_{1} X$ is weakly equivalent to $J^{*} L_{2} J_{*} X$.

Proof. First note that $X \simeq \operatorname{hocolim}_{\Delta^{o p}}\left([n] \rightarrow \coprod_{i} \Delta\left[n_{i}\right]^{t}\right)$ where the values of $i$ depend on $n$. We begin by looking at $L_{1} X$. Using Lemma 4.1 we have the following:

$$
\begin{aligned}
L_{1} X & \simeq L_{1} \operatorname{hocolim}_{\Delta}{ }^{o p}\left([n] \mapsto \coprod \Delta\left[n_{i}\right]_{*}^{t}\right) \\
& \simeq L_{1} \operatorname{hocolim}_{\Delta^{o p}} L_{1}\left([n] \mapsto \coprod \Delta\left[n_{i}\right]_{*}^{t}\right) \\
& \simeq L_{1} \operatorname{hocolim}_{\Delta^{o p}}\left([n] \mapsto \operatorname{nerve}\left(T_{\sum n_{i}}\right)^{t}\right)
\end{aligned}
$$

However, hocolim $\Delta^{o p}\left([n] \mapsto \operatorname{nerve}\left(T_{\sum n_{i}}\right)^{t}\right)$ is already local, a fact which follows from the fact that the homotopy colimit can be taken at each level, yielding a Segal precategory in $\mathcal{L} \mathcal{S} S p_{*, c}$ which is still a Segal category. 
Working from the other side of the desired equation, we obtain, using the fact that left adjoints commute with colimits and homotopy colimits:

$$
\begin{aligned}
J^{*} L_{2} J_{*} X & \simeq J^{*} L_{2} J_{*} \operatorname{hocolim}_{\Delta^{o p}}\left([n] \mapsto \coprod \Delta\left[n_{i}\right]_{*}^{t}\right) \\
& \simeq J^{*} L_{2} \operatorname{hocolim}_{\Delta^{o p}} J_{*}\left([n] \mapsto \coprod \Delta\left[n_{i}\right]_{*}^{t}\right) \\
& \simeq J^{*} L_{2} \operatorname{hocolim}_{\Delta^{o p}} L_{2} J_{*}\left([n] \mapsto \coprod \Delta\left[n_{i}\right]_{*}^{t}\right) \\
& \simeq J^{*} L_{2} \operatorname{hocolim}_{\Delta^{o p}}\left([n] \mapsto M\left[\sum n_{i}\right]\right)
\end{aligned}
$$

In $\mathcal{T}_{M}$, this looks like the diagram in $\boldsymbol{\Delta}^{o p}$, but now we have maps in the theory of monoids rather than simplicial maps. Thus, the restriction map $J_{*}$ will give the same objects but with simplicial maps, as wished.

We begin by showing that we have a Quillen pair even before we apply the localization functors. Notice that we return to using our model structure $\mathcal{S} S p_{*, f}$ with levelwise fibrations.

Proposition 4.8. The adjoint pair

$$
J_{*}: \mathcal{S} S p_{*, f} \rightleftarrows \mathcal{S} \mathcal{S e t}_{*}^{\mathcal{T}_{M}}: J^{*}
$$

is a Quillen pair.

Proof. In each case the fibrations and weak equivalences are defined levelwise. Since the right adjoint $J^{*}$ preserves the spaces at each level, it must preserve both fibrations and acyclic fibrations.

We then need to show that this Quillen pair induces a Quillen equivalence between the localized model category structures in order to prove our main theorem. While we will be working with $\mathcal{L} \mathcal{S} \mathcal{S} p_{*, f}$ rather than $\mathcal{L} \mathcal{S} \mathcal{S} p_{*, c}$, the calculations we made above will still hold because the weak equivalences are the same in each case (see the end of section 3 ).

Proof of Theorem 1.7. We first need to show that we still have a Quillen pair even after localizing each category. This fact follows from Theorem 2.7 after we notice that the left derived localizing set $\mathbf{L} J_{*} \varphi[13,8.5 .11]$ is the same as the set of maps $S=\left\{p_{0}, p_{1}, \ldots\right\}$ that we localize with respect to in order to obtain $\mathcal{L S S E}_{e} s_{*}^{\mathcal{T}_{M}}$, as follows. Consider the maps $\varphi_{k}: \coprod_{k} \Delta[1]_{*}^{t} \rightarrow \Delta[k]_{*}^{t}$ for each $k$. If we localize this map, we obtain $\coprod_{k}$ nerve $\left(T_{1}\right)^{t} \rightarrow$ nerve $\left(T_{k}\right)^{t}$, where $T_{k}$ denotes the free monoid on $k$ generators. Then we can apply the functor $J_{*}$ to obtain a map

$$
J_{*}\left(\coprod_{k} \operatorname{nerve}\left(T_{1}\right)^{t}\right) \rightarrow J_{*}\left(\operatorname{nerve}\left(T_{k}\right)^{t}\right)
$$

which we can then localize to obtain, by Lemma 4.6 the map $\coprod_{k} M[1] \rightarrow M[k]$. However, this is precisely the map $p_{k}: \coprod_{k} \operatorname{Hom}\left(T_{1},-\right) \rightarrow \operatorname{Hom}\left(T_{k},-\right)$.

We now need to show that we have a Quillen equivalence. First, we need to know that the right adjoint $J^{*}$ reflects weak equivalences between fibrant objects. In each of the two localized model categories $\mathcal{L} \mathcal{S} \mathcal{S} p_{*, f}$ and $\mathcal{L} \mathcal{S} \mathcal{S} e t s_{*}^{\mathcal{T}_{M}}$, an object is fibrant if and only if it is local and fibrant in the unlocalized model category. Therefore, in each case a weak equivalence between fibrant objects is a levelwise weak equivalence. Since $J^{*}$ does not change the spaces at each level, it must reflect weak equivalences between fibrant objects. 
Finally, by Proposition $4.7 L_{1} X \simeq J^{*} L_{2} J_{*} X$ for any functor $X: \boldsymbol{\Delta}^{o p} \rightarrow$ SSets, and in particular for any cofibrant $X$.

Notice that our Quillen equivalences compose:

$$
\mathcal{L S S} p_{*, f} \rightleftarrows \mathcal{L S S E} s_{*}^{\mathcal{T}_{M}} \rightleftarrows \mathcal{L S S e t s}^{\mathcal{T}_{M}} \rightleftarrows \mathcal{A l g}{ }^{\mathcal{T}_{M}}
$$

(where the left adjoint functors are the topmost maps). Therefore, we actually have a single Quillen equivalence

$$
\mathcal{L} \mathcal{S} S p_{*, f} \rightleftarrows \mathcal{A l g}{ }^{\mathcal{T}_{M}} .
$$

\section{A Generalization to Simplicial Categories and Segal Categories}

We would like to generalize our result to the category of simplicial categories which have a set $\mathcal{O}$ of objects, where the morphisms are required to be the identity on this set, since a simplicial category is a generalization of a simplicial monoid.

Definition 5.1. A simplicial category is a category enriched over simplicial sets, i.e. a category in which the morphisms between any two objects form a simplicial set.

This use of the term "simplicial category" is potentially confusing. We do not mean that a simplicial category is simply a simplicial object in the category of categories. It is actually a simplicial object in the category of categories in which the categories at each level have the same objects. In particular, the face and degeneracy maps are the identity on the objects.

Analogously, we will generalize to Segal categories with the same set $\mathcal{O}$ in degree zero. As we have seen, any Segal category can be described as a functor from the simplicial category $\boldsymbol{\Delta}^{o p}$ to the category SSets of simplicial sets satisfying two conditions: discreteness of the zero space and a product condition up to homotopy (Definition 1.2). However, we will find it more convenient, when dealing with Segal categories with a fixed object set, to use a larger indexing category which specifies the objects.

Let $\mathcal{O}$ be a set. We define the category $\boldsymbol{\Delta}_{\mathcal{O}}^{o p}$ as follows. The objects are given by $[n]_{x_{0}, \ldots, x_{n}}$ where $n \geq 0$ and $\left(x_{0}, \ldots x_{n}\right) \in \mathcal{O}^{n+1}$. The $[n]$ should be thought of as in the simplicial category $\boldsymbol{\Delta}^{o p}$; however, recall that when we work with Segal categories we will require all morphisms to preserve the objects. Therefore, we need to have a separate $[n]$ for each possible $(n+1)$-tuple of objects in $\mathcal{O}$. The morphisms in $\boldsymbol{\Delta}_{\mathcal{O}}^{o p}$ are those of $\boldsymbol{\Delta}^{o p}$ but depend on the choice of $\left(x_{0}, \ldots, x_{n}\right)$. Specifically, the face maps are

$$
d_{i}:[n]_{x_{0}, \ldots, x_{n}} \rightarrow[n-1]_{x_{0}, \ldots, \widehat{x}_{i}, \ldots, x_{n}}
$$

and the degeneracy maps are

$$
s_{i}:[n]_{x_{0}, \ldots, x_{n}} \rightarrow[n+1]_{x_{0}, \ldots, x_{i-1}, x_{i}, x_{i}, x_{i+1}, \ldots, x_{n}} .
$$

We will sometimes refer to our Segal precategories with $\mathcal{O}$ in degree zero as $\boldsymbol{\Delta}_{\mathcal{O}}^{o p}$ spaces. Note that if $\mathcal{O}$ is the one-object set, then $\boldsymbol{\Delta}_{\mathcal{O}}^{o p}$ is just $\boldsymbol{\Delta}^{o p}$.

Now we can use this notation to describe Segal categories.

Definition 5.2. Given a category $\mathcal{C}$, a functor $F: \boldsymbol{\Delta}_{\mathcal{O}}^{o p} \rightarrow \mathcal{C}$ is $\mathcal{O}$-special if for each $n \geq 2$ the map

$$
F\left([n]_{x_{0}, \ldots, x_{n}}\right) \stackrel{\simeq}{\longrightarrow} F\left([1]_{x_{0}, x_{1}}\right) \times_{F[0]_{x_{1}}} \cdots \times_{F[0]_{x_{n-1}}} F\left([1]_{x_{n-1}, x_{n}}\right)
$$

is a weak equivalence of simplicial sets. 
Now, we can think of a Segal category with $\mathcal{O}$ in dimension zero as an $\mathcal{O}$-special functor $\boldsymbol{\Delta}_{\mathcal{O}}^{o p} \rightarrow$ SSets. Recall from section 3 that we have model category structures $\mathcal{L} \mathcal{S} \mathcal{S} p_{\mathcal{O}, f}$ and $\mathcal{L} \mathcal{S} \mathcal{S} p_{\mathcal{O}, c}$ on the category of Segal precategories with $\mathcal{O}$ in degree zero, in each of which the fibrant objects are Segal categories.

We would like to think of the category of simplicial categories with object set $\mathcal{O}$ as a diagram category as well. To do so, we need to define the notion of a multi-sorted algebraic theory. To see more details, see 4 .

Definition 5.3. Given a set $S$, an $S$-sorted algebraic theory (or multi-sorted theory) $\mathcal{T}$ is a small category with objects $T_{\underline{\alpha}^{n}}$ where $\underline{\alpha}^{n}=\left\langle\alpha_{1}, \ldots, \alpha_{n}>\right.$ for $\alpha_{i} \in S$ and $n \geq 0$ varying, and such that each $T_{\underline{\alpha}^{n}}$ is equipped with an isomorphism

$$
T_{\underline{\alpha}^{n}} \cong \prod_{i=1}^{n} T_{\alpha_{i}} .
$$

For a particular $\underline{\alpha}^{n}$, the entries $\alpha_{i}$ can repeat, but they are not ordered. There exists a terminal object $T_{0}$ (corresponding to the empty object of $S$ ).

In particular, we can talk about the theory of $\mathcal{O}$-categories, which we will denote by $\mathcal{T}_{\mathcal{O} \text { C } a t}$. To define this theory, first consider the category $\mathcal{O} \mathcal{C}$ at whose objects are the categories with a fixed object set $\mathcal{O}$ and whose morphisms are the functors which are the identity map on the objects. The objects of $\mathcal{T}_{\mathcal{O} \text { e } a t}$ are categories which are freely generated by directed graphs with vertices corresponding to the elements of the set $\mathcal{O}$. This theory will be sorted by pairs of elements in $\mathcal{O}$, corresponding to the morphisms with source the first element and target the second. In other words, this theory is $(\mathcal{O} \times \mathcal{O})$-sorted [4, 3.5]. (In the one-object case, we have the ordinary theory of monoids.) We can then say that a simplicial category with object set $\mathcal{O}$ is a strict $\mathcal{T}_{\mathcal{O} \text { C } a t}$-algebra, where strict and homotopy $\mathcal{T}$-algebras are defined for multi-sorted theories $\mathcal{T}$ just as for ordinary algebraic theories.

Again, we have a model structure $\mathcal{A l} g^{\mathcal{T}}$ on the category of all $\mathcal{T}$-algebras and a model structure $\mathcal{S} \mathcal{S}_{e} \boldsymbol{s}^{\mathcal{T}}$ on the category of all functors $\mathcal{T} \rightarrow \mathcal{S}$ Sets which can be localized as before to obtain a model category structure $\mathcal{L} \mathcal{S} \mathcal{S}$ et ${ }^{\mathcal{T}}{ }^{\mathcal{S}}$ in which the local objects are homotopy $\mathcal{T}_{\text {-algebras }}$ 4]. For $\mathcal{T}_{\mathcal{O}}$ eat , we can define a category $\mathcal{S} \mathcal{S} e t \boldsymbol{S}_{\mathcal{O}}^{\mathcal{T}_{\text {Oe }} \text { et }}$ of functors $\mathcal{T}_{\mathcal{O} \text { Cat }} \rightarrow$ SSets which send $T_{0}$ to $\coprod_{\mathcal{O}} \Delta[0]$. Making modifications as in the case of $\mathcal{L} \mathcal{S} \mathcal{S} e t s_{*}^{\mathcal{T}_{M}}$, we can define a model structure $\mathcal{L} \mathcal{S} \mathcal{S} e t s_{\mathcal{O}}^{\mathcal{T}_{\mathcal{O}} \text { eat }}$ which is Quillen equivalent to $\mathcal{L S S}$ ets $^{\mathcal{T}_{\mathcal{O}} \text { eat }}$.

Furthermore, Badzioch's rigidification theorem 3.4 for algebraic theories can be generalized to the case of multi-sorted theories, and therefore we have that there is a Quillen equivalence of model categories between $\mathcal{A} l g^{\mathcal{T}}$ and $\mathcal{L} \mathcal{S} S e t s^{\mathcal{T}}$ for any multisorted theory $\mathcal{T}$ [4 5.1]. Applying this result to the multi-sorted theory $\mathcal{T}_{\mathcal{O}} \mathcal{C}_{a t}$ and using the Quillen equivalence in the previous paragraph, we have reduced the problem to finding a Quillen equivalence between $\mathcal{L} \mathcal{S} \mathcal{S} p_{\mathcal{O}, f}$ and $\mathcal{L} \mathcal{S} \mathcal{S} e t s_{\mathcal{O}}^{\mathcal{T}_{\mathcal{O}} \text { eat }}$. We begin as before by defining a map of diagrams $J: \boldsymbol{\Delta}_{\mathcal{O}}^{o p} \rightarrow \mathcal{T}_{\mathcal{O} \text { e at }}$ which then induces a map $J^{*}: \mathcal{S S e t}_{S_{\mathcal{O}}}^{\mathcal{T}_{\mathcal{O}} \text { eat }} \rightarrow \mathcal{S S e t}^{\boldsymbol{\Delta}_{\mathcal{O}}^{o p}}$.

First consider an arbitrary category $\mathcal{C}$ with object set $\mathcal{O}$. Then its nerve will be a Segal category with the set $\mathcal{O}$ in dimension zero. Since it is a Segal category, we can view it as an $\mathcal{O}$-special functor $\boldsymbol{\Delta}_{\mathcal{O}}^{o p} \rightarrow$ SSets with the specification that $[0]_{x_{i}} \mapsto x_{i}$ for any $x_{i} \in \mathcal{O}$.

Now, given an integer $n \geq 0$ and $\underline{x}=\left(x_{0}, \ldots, x_{n}\right) \in \mathcal{O}^{n+1}$, define $T_{n, \underline{x}}$ to be the free category with object set $\mathcal{O}$ and morphisms freely generated by the set 
$\left\{x_{i-1} \rightarrow x_{i} \mid 1 \leq i \leq n\right\}$. However, note that this free category is, in light of the definition of multi-sorted theory, just the object $T_{\underline{\alpha}}$ where $\underline{\alpha}=\left(\alpha_{1}, \ldots, \alpha_{n}\right)$ and $\alpha_{i}=\left(x_{i-1}, x_{i}\right) \in \mathcal{O} \times \mathcal{O}$. (We will find both notations useful.)

We then obtain a simplicial diagram as follows:

$$
\operatorname{Hom}\left(T_{0}, \mathcal{C}\right) \Leftarrow \coprod_{\alpha_{1}} \operatorname{Hom}\left(T_{\alpha_{1}}, \mathcal{C}\right) \Leftarrow \coprod_{\underline{\alpha}=\left(\alpha_{1}, \alpha_{2}\right)} \operatorname{Hom}\left(T_{\underline{\alpha}}, \mathcal{C}\right) \cdots
$$

Because we have a coproduct at each level, this diagram is not representable by a cosimplicial diagram. However, if we split up the coproducts and consider it as a diagram of objects $\operatorname{Hom}\left(T_{\underline{\alpha}}, \mathcal{C}\right)$ varying over $\underline{\alpha}$ and $n$, we get a map $J: \boldsymbol{\Delta}_{\mathcal{O}}^{o p} \rightarrow$ $\mathcal{T}_{\mathcal{O} \text { Cat }}$. Then we define the map

$$
J^{*}: \operatorname{SSets}_{\mathcal{O}}^{\mathcal{T}_{\mathcal{O} e a t}} \rightarrow \operatorname{SSets}^{\boldsymbol{\Delta}_{\mathcal{O}}^{o p}}
$$

to be the one induced by this map $J$ above. Putting our model structures on these categories we get

$$
J^{*}: \mathcal{L S S E t s}_{\mathcal{O}}^{\mathcal{J}_{\mathcal{O}} a t} \rightarrow \mathcal{L} \mathcal{S S S} p_{\mathcal{O}, f} .
$$

We will denote by $L_{1}$ the localization of simplicial spaces with $\mathcal{O}$ in dimension zero, namely the functorial fibrant replacement functor in $\mathcal{L} \mathcal{S} S p_{\mathcal{O}, c}$. (As above, we will make our calculations in this category rather than in $\left.\mathcal{L} S \mathcal{S} p_{\mathcal{O}, f}.\right)$ We have an analogous localization in the category $\mathcal{L S S e t s}_{\mathcal{O}}^{\mathcal{T}_{\mathcal{O}} \text { eat }}$, which we will denote by $L_{2}$.

From there, we can apply the methods used in the previous section. We first consider what happens when we localize an $n$-simplex $\Delta[n]_{x_{0}, \ldots, x_{n}}$. If $x_{i}=x_{j}$ for all $0 \leq i, j \leq n$ then we get the free monoid on $n$ generators, with object $x_{i}$. However, if $x_{i} \neq x_{j}$ for some $0 \leq i, j \leq n$, we get the nerve of the free category generated by the directed graph

$$
x_{0} \rightarrow x_{1} \rightarrow \cdots \rightarrow x_{n}
$$

For example, if $x_{i} \neq x_{j}$ for all $0 \leq i, j \leq n$, then the localization only consists of including in composites, say 1-simplices $x_{i} \rightarrow x_{i+2}$. If $n=1$ and $x_{0} \neq x_{1}$, then the localization does not change the Segal category since there are no "composable" 1 -simplices. We will denote the free category by $T_{n, \underline{x}}$, as before, and its nerve by $G_{n, \underline{x}}=\operatorname{nerve}\left(T_{n, \underline{x}}\right)^{t}$.

Now, define $J_{*}$ be the left Kan extension of the map $J^{*}$. However, note that again we must apply the localization functor $L_{2}$ to assure that we get a homotopy $\mathcal{T}_{\mathcal{O} \text { e } a t}$-algebra in $\mathcal{L S S e t s}_{\mathcal{O}}^{\mathcal{J}_{\mathcal{O}} \text { e at }}$. As before, it suffices to show that for any $\boldsymbol{\Delta}_{\mathcal{O}}^{o p}$-space $X$, we have that $L_{1} X=J^{*} L_{2} J_{*} X$. We will first need a lemma.

Analogous to $M[k]$ above, define $C[n]_{\underline{x}}$ to be the $\mathcal{T}_{\mathcal{O}}$ Cat $_{\text {-diagram in of simplicial }}$ sets which has the same simplicial set at each level as $G_{n, \underline{x}}$, but with theory maps between them rather than the simplicial maps.

Lemma 5.4. In $\mathcal{L S S E}$ ts $_{\mathcal{O}}^{\mathcal{T}_{\text {Oe at }}}, L_{2} J_{*}\left(G_{n, \underline{x}}\right)$ is weakly equivalent to $C[n]_{\underline{x}}$.

Proof. It suffices to show that for a local object $X$ in $\mathcal{L S S e t}_{\mathcal{O}}^{\mathcal{T}_{\mathcal{O}} \text { eat }}$, we have that

$$
\operatorname{Hom}_{\mathcal{L S S e t s}_{\mathcal{O}}^{\mathcal{J}_{\mathcal{O C}} \text { et }}}\left(L_{2} J_{*}\left(G_{n, \underline{x}}\right), X\right) \simeq X\left(T_{n, \underline{x}}\right) .
$$


This can be proved as follows:

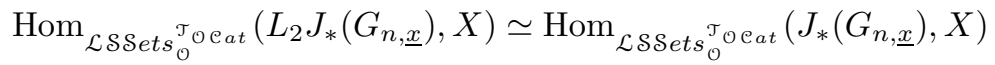

$$
\begin{aligned}
& \simeq \operatorname{Hom}_{\mathcal{L S S} p_{\mathcal{O}, c}}\left(G_{n, \underline{x}}, J^{*} X\right) \\
& \simeq \operatorname{Hom}_{\mathcal{L S S} p_{\mathcal{O}, c}}\left(L_{1} \Delta[n]_{\underline{x}}, J^{*} X\right) \\
& \simeq \operatorname{Hom}_{\mathcal{L S S} p_{\mathcal{O}, c}}\left(\Delta[n]_{\underline{x}}, J^{*} X\right) \\
& \simeq J^{*}(X)[n]_{\underline{x}} \\
& \simeq X\left(T_{n, \underline{x}}\right)
\end{aligned}
$$

Note that in $\mathcal{T}_{\mathcal{O C} \text { eat }}$, we would usually write $T_{\underline{\alpha}}$ instead of $T_{n, \underline{x}}$, where $\underline{\alpha}=\left(\alpha_{1}, \ldots, \alpha_{n}\right)$ and $\alpha_{i}=\left(x_{i-1}, x_{i}\right)$ for $1 \leq i \leq n$.

Now, using this lemma, the following proposition suffices to prove the theorem.

Proposition 5.5. Given any $\boldsymbol{\Delta}_{\mathcal{O}}^{o p}$-space $X, L_{1} X$ and $J^{*} L_{2} J_{*} X$ are weakly equivalent.

Proof. Given any $\boldsymbol{\Delta}_{\mathcal{O}}^{o p}$-space $X$, it can be written as the homotopy colimit

$$
X \simeq \operatorname{hocolim}_{\Delta}{ }^{o p}\left([n] \mapsto \amalg_{n_{i}, \underline{x}} \Delta\left[n_{i}\right]_{\underline{x}}\right)
$$

where the $n_{i}$ depend on $n$ and $\underline{x}=\left(x_{1}, \ldots, x_{n_{i}}\right)$. We then have the following:

$$
\begin{aligned}
L_{1} X & \simeq L_{1} \operatorname{hocolim}_{\Delta^{o p}}\left([n] \mapsto \amalg_{n_{i}, \underline{x}} \Delta\left[n_{i}\right]_{\underline{x}}\right) \\
& \simeq L_{1} \operatorname{hocolim}_{\boldsymbol{\Delta}^{o p}} L_{1}\left([n] \mapsto \amalg_{n_{i}, \underline{x}} \Delta\left[n_{i}\right]_{\underline{x}}\right) \\
& \simeq L_{1} \operatorname{hocolim}_{\boldsymbol{\Delta}^{o p}}\left([n] \mapsto \amalg_{n_{i}, \underline{x}} G_{n_{i}, \underline{x}}\right)
\end{aligned}
$$

Working from the other side, we have

$$
\begin{aligned}
J^{*} L_{2} J_{*} X & \simeq J^{*} L_{2} J_{*}^{h} \operatorname{hocolim}_{\Delta^{o p}}\left([n] \mapsto \amalg_{n_{i}, \underline{x}} \Delta\left[n_{i}\right]_{\underline{x}}\right) \\
& \simeq J^{*} L_{2} \operatorname{hocolim}_{\boldsymbol{\Delta}^{o p}} J_{*}\left([n] \mapsto \amalg_{n_{i}, \underline{x}} \Delta\left[n_{i}\right]_{\underline{x}}\right) \\
& \simeq J^{*} L_{2} \operatorname{hocolim}_{\boldsymbol{\Delta}^{o p}} L_{2} J_{*}\left([n] \mapsto \amalg_{n_{i}, \underline{x}} \Delta\left[n_{i}\right]_{\underline{x}}\right) \\
& \simeq J^{*} L_{2} \operatorname{hocolim}_{\boldsymbol{\Delta}^{o p}}\left([n] \mapsto \amalg_{n_{i}, \underline{x}} C\left[n_{i}\right]_{\underline{x}}\right)
\end{aligned}
$$

But these two are equal, by the above lemma.

Now, using the same arguments as in the last section, we can prove the following theorem:

Theorem 5.6. The adjoint pair

$$
J_{*}: \mathcal{L S S S} p_{\mathcal{O}, f} \rightleftarrows \mathcal{L} \mathcal{S S e t s}_{\mathcal{O}}^{\mathcal{T}_{\mathcal{O}} \text { e at }}: J^{*}
$$

is a Quillen equivalence.

In particular, composing with the Quillen equivalences given by the generalizations of Theorem 3.4 and Proposition 3.12 there is a Quillen equivalence

$$
\mathcal{L S S} p_{\mathcal{O}, f} \rightleftarrows \mathcal{A l g}{ }^{\mathcal{T}_{\mathcal{O} e a t}} .
$$




\section{REFERENCES}

[1] Bernard Badzioch, Algebraic theories in homotopy theory, Ann. of Math. (2) 155 (2002), no. 3, 895-913.

[2] Bernard Badzioch, Kuerak Chung, and Alexander A. Voronov, Yet another delooping machine, preprint available at math.AT/0403098

[3] J.E. Bergner, A model category structure on the category of simplicial categories, preprint available at math.AT/0406507 to appear in Trans. of the AMS.

[4] J.E. Bergner, Multi-sorted algebraic theories, preprint available at math.AT/0508152

[5] J.E. Bergner, Three models for the homotopy theory of homotopy theories, preprint available at math.AT $/ 0504334$

[6] Francis Borceux, Handbook of Categorical Algebra, Volume 2, Encyclopedia of Mathematics and its Applications 51, Cambridge University Press, Cambridge, 1994.

[7] A.K. Bousfield, The simplicial homotopy theory of iterated loop spaces, unpublished manuscript.

[8] W.G. Dwyer and D.M. Kan, Simplicial localizations of categories, J. Pure Appl. Algebra 17 (1980), no. 3, 267-284.

[9] W.G. Dwyer and D.M. Kan, Function complexes in homotopical algebra, Topology 19 (1980), 427-440.

[10] W.G. Dwyer and D.M. Kan, Equivalences between homotopy theories of diagrams, Algebraic topology and algebraic K-theory (Princeton, N.J., 1983), 180-205, Ann. of Math. Stud., 113, Princeton Univ. Press, Princeton, NJ, 1987.

[11] W.G. Dwyer and J. Spalinski, Homotopy theories and model categories, in Handbook of Algebraic Topology, Elsevier, 1995.

[12] P.G. Goerss and J.F. Jardine, Simplicial Homotopy Theory, Progress in Math, vol. 174, Birkhauser, 1999.

[13] Philip S. Hirschhorn, Model Categories and Their Localizations, Mathematical Surveys and Monographs 99, AMS, 2003.

[14] A. Hirschowitz and C. Simpson, Descente pour les $n$-champs, preprint available at math.AG/9807049

[15] Mark Hovey, Model Categories, Mathematical Surveys and Monographs 63, AMS, 1999.

[16] Saunders MacLane, Categories for the Working Mathematician, Second Edition, Graduate Texts in Mathematics 5, Springer-Verlag, 1997.

[17] F. William Lawvere, Functorial semantics of algebraic theories, Proc. Nat. Acad. Sci U.S.A. 50(1963) 869-872.

[18] Daniel Quillen, Homotopical Algebra, Lecture Notes in Math 43, Springer-Verlag, 1967.

[19] Charles Rezk, A model for the homotopy theory of homotopy theory, Trans. of the AMS, 353(3), 973-1007.

[20] Stefan Schwede, Stable homotopy of algebraic theories, Topology 40 (2001), 1-41.

[21] Graeme Segal, Categories and cohomology theories, Topology 13 (1974), 293-312.

[22] J.D. Stasheff, Homotopy associativity of $H$-spaces I, Trans. Amer. Math. Soc. 108 (1963), 275-292.

[23] R.W. Thomason, Uniqueness of delooping machines, Duke Math. J. 46 (1979), no. 2, 217-252.

Kansas State University, 138 Cardwell Hall, Manhattan, KS 66506

E-mail address: bergnerj@member.ams.org 